\title{
OnionMHC: A Deep Learning Model for Peptide - HLA-A*02:01 Binding Predictions using both Structure and Sequence Feature Sets
}

\section{Shikhar Saxena}

Nanyang Technological University

Sambhavi Animesh

National University of Singapore

Melissa Fullwood

Nanyang Technological University

Yuguang Mu ( $\nabla$ ygmu@ntu.edu.sg )

Nanyang Technological University

\section{Research Article}

Keywords: Peptide-MHC binding, Structure, Sequence, Binding Affinity, Deep Learning

Posted Date: December 15th, 2020

DOl: https://doi.org/10.21203/rs.3.rs-124695/v1

License: (1) This work is licensed under a Creative Commons Attribution 4.0 International License. Read Full License 


\section{OnionMHC: a deep learning model for peptide - HLA-A*02:01 binding}

2 predictions using both structure and sequence feature sets

3 Shikhar Saxena ${ }^{1}$, Sambhavi Animesh², Melissa J. Fullwood ${ }^{1,2}$, and Yuguang Mu*1

41 School of Biological Sciences, Nanyang Technological University, 60 Nanyang Drive,

5 Singapore, 637551

62 Cancer Science Institute, National University of Singapore, 14 Medical Drive, Singapore, 7117599.

$8 \quad *$ Corresponding author: ygmu@ntu.edu.sg

\section{Abstract:}

\section{Background:}

12 The peptide binding to Major Histocompatibility Complex (MHC) proteins is an important step in the

13 antigen-presentation pathway. Thus, predicting the binding potential of peptides with MHC is essential

14 for the design of peptide-based therapeutics. Most of the available machine learning-based models

15 predict the peptide-MHC binding based on the sequence of amino acids alone. Given the importance of

16 structural information in determining the stability of the complex, here we have utilized both the complex

17 structure and the peptide sequence features to predict the binding affinity of peptides to human receptor

18 HLA-A*02:01. To our knowledge, no such model has been developed for the human HLA receptor before

19 that incorporates both structure and sequence-based features.

\section{Results:}

21 We have applied machine learning techniques through the natural language processing (NLP) and

22 convolutional neural network to design a model that performs comparably with the existing state-of-the-

23 art models. Our model shows that the information from both sequence and structure domains results in

24 enhanced performance in the binding prediction compared to the information from one domain alone.

25 The testing results in 18 weekly benchmark datasets provided by the Immune Epitope Database (IEDB) as 
26 well as experimentally validated peptides from the whole-exome sequencing analysis of the breast cancer

27 patients indicate that our model has achieved state-of-the-art performance.

\section{Conclusion:}

29 We have developed a deep-learning model (OnionMHC) that incorporates both structure as well as 30 sequence-based features to predict the binding affinity of peptides with human receptor HLA-A*02:01.

31 The model demonstrates state-of-the-art performance on the IEDB benchmark dataset as well as the 32 experimentally validated peptides. The model can be used in the screening of potential neo-epitopes for 33 the development of cancer vaccines or designing peptides for peptide-based therapeutics. OnionMHC is 34 freely available at https://github.com/shikhar249/OnionMHC

36 Keywords: Peptide-MHC binding; Structure; Sequence; Binding Affinity; Deep Learning

\section{Background:}

39 Neo-epitopes are the unique peptide signatures produced by cancer cells, some of which are presented

40 on their outer surface and recognized by T cells [1, 2]. For the successful presentation of these antigenic

41 peptides, the peptide should be able to bind to the Major Histocompatibility Complex (MHC) receptor

42 with enough strength $\left(\mathrm{IC}_{50}<500 \mathrm{nM}\right)$ in most cases. This makes peptide binding to $\mathrm{MHC}$ the most crucial

43 and selective step in antigen presentation[3]. Thus, there is a need to develop computational algorithms

44 that can accurately predict the binding affinity of peptides to MHC.

45 There have been several approaches made in this regard, mainly in terms of allele-specific or pan-specific

46 models. In allele-specific models, the model was trained on a specific allele[4] whereas in a pan-specific

47 version the model was trained on multiple alleles data [5]. These models were mainly based on either the

48 sequence-based features[5, 6] or the structure-based features[7, 8]. Generally, most of the previous 
49 approaches employed the sequence-based features for predicting the binding affinity. Different neural 50 network models like convolutional neural network models $[6,9,10]$, attention-based neural network 51 models [11] have been employed with different ways of sequence information encoding. Other studies 52 have used the structure-based features only for predicting the binding affinity [7, 12]. The information 53 provided by the peptide-MHC 3D structures can potentially bring to light yet unknown drivers of T-cell 54 activation thus, facilitating more accurate predictions. However, this direction is progressing at a very 55 slow pace, primarily because 1) a very limited number of experimentally determined peptide-MHC 56 complex structures are available; and 2) the structural modeling is computationally expensive, rendering 57 it feasible only on a small scale. To our knowledge, there is no such model that combines both structure 58 and sequence-based features to predict the binding affinity of peptides with human MHC receptors. 59 Recently a study demonstrated the potential of combined structure and sequence feature sets in 60 predicting the binding affinity of peptides to mouse allele $\mathrm{H}-2^{\mathrm{D}}$ [13]. However, this study used a very 61 simple machine learning algorithm of the support vector machines which is not sophisticated enough to 62 capture all the intrinsically complex patterns. Furthermore, the authors have not made the model publicly 63 available, so it is difficult to compare with their model.

64 In this study, we have used both complex structure and sequence feature sets to predict the binding 65 affinity of the peptides to widely studied human HLA-A*02:01 alleles. The structural features we used are 66 the contact pairs between the atoms in the peptide and the MHC receptor. The contact pairs based 67 structural features are known to perform well for the small molecule and protein complex in the PDBbind 68 dataset[14]. Here, the similar contact-pairs based structural features for the peptide-MHC complex were 69 combined with sequence-based features. Different encodings of raw peptide sequences and different 70 architectures, such as Long Short-Term Memory (LSTM) based neural network models, were tested. Our 71 model's performance is comparable and even slightly better than the current state-of-the-art model 72 NetMHCpan4.0[5], HLAthena [15], and the convolutional neural network model ConvMHC [9] on the 
73 independent IEDB benchmark dataset. All these models are the sequence-based models that take solely

74 the sequence of peptides to make the binding affinity predictions. We have also performed molecular

75 dynamics simulations to demonstrate the stability of the predicted models. Moreover, our model has

76 been used to predict the binding affinity of the peptides derived from the neoantigen analysis of the

77 exome sequencing samples from the breast cancer patients which were further validated experimentally.

78 The exome sequencing and neoantigen analysis will be reported in an upcoming manuscript.

79 OnionMHC is publicly available and can be accessed at https://github.com/shikhar249/OnionMHC

80

\section{Methods:}

\section{Dataset collection}

83 The peptide sequences and their binding affinities to HLA-A*02:01 were downloaded from IEDB

84 [16](http://tools.iedb.org/main/datasets/). This dataset was compiled by combining BD2009, BD2013, 85 and the blind data [17] and it also included additional data that was not publicly available at that time.

86 The detailed dataset used for the training is provided in Table S3, Additional File 4. Although the HLA87 A*02:01 receptors can bind to peptides of varying lengths, we only considered 9 mers for this study as 9 88 mers are the most preferred binders to HLA-A*02:01. The dataset contains 9051 peptides of length 9 89 residues with their corresponding $I C_{50}$ values with $H L A-A^{*} 02: 01$ receptors. The peptides with $I C_{50}$ less than $90500 \mathrm{nM}$ were considered as the positive binders rendering 3273 peptides as positive and 5778 peptides

91 as negative towards binding to HLA-A*02:01. The same dataset from IEDB was utilized to train several 92 other peptide-MHC binding affinity predicting models[5, 9-11, 18, 19], thus making comparison 93 straightforward. The comparison of these binding affinity predicting models was done on the weekly 94 benchmark data provided by IEDB (http://tools.iedb.org/auto bench/mhci/weekly/) which is different 95 from the dataset used for training.

\section{Structure Modelling}


97 The structure of HLA-A*02:01 with the template peptide backbone of 9 residues was taken from

98 PDB[20](PDB ID: 1AKJ). The nonameric peptides from PDB structures show high conservation in their

99 backbones[7]. The side chains of different peptides were modeled on the same template structure using

100 SCWRL4 software[21]. It has been shown that SCWRL4 can reproduce experimentally determined X-ray

101 structures of peptide - HLA complexes with the lowest root mean square deviation[22]. The structures

102 obtained from SCWRL4 were further energy minimized with the default settings in GROMACS[23] to take

103 care of any bad contacts thus, generating atomic-level structural models for different peptides with HLA-

$104 A^{*} 02: 01$. This approach of structure generation from peptide sequences is similar to the one used in a

105 previously published study [7].

\section{Structure feature generation: contact pair counts}

107 In this study, the structure features are characterized by the contact pair counts between the peptide

108 (ligand) and the HLA receptor (protein). The structure-based features are adapted from a recent study

109 characterizing the binding of small molecule ligands with protein receptors [14]. In this case, each protein

110 atom or ligand atom can be categorized into one of the 8 element types:

$$
E_{L}=\left[C_{\text {aliphatic }}, C_{\text {aromatic }}, O_{\text {carbonyl }}, O_{\text {hydroxyl }}, N, H, S, D u\right]
$$

111 which would generate a total of 64 ( $\left.8_{\text {ligand }} \times 8_{\text {protein }}\right)$ element-pair combinations between the ligand and

112 the protein (Du represents all other remaining elements). Here, we defined 60 shells of width $\sim 0.5 \AA$

113 around each ligand atom and counted the number of different protein atoms within each of these 60

114 shells. The center of the first shell was set at the distance of $1 \AA$ from the ligand atom and the center of

115 the last shell has a distance of $30.5 \AA$ to the ligand atom. Such a broad distance range covers both local

116 and non-local interaction information between the ligand and the protein.

117 The atom-based contact number information between the ligand and the protein was then converted

118 into element-pair specific and distance shell specific contact numbers. This was done by summing up all 
119 the contact numbers between the ligand atoms and the protein atoms for a given elemental pair in a

120 given shell resulting in 64 element-pair specific contact numbers for each of the 60 shells.

121 Thus, one peptide-HLA complex can be represented by an image matrix of the size $(60 \times 64)$ where 60 is

122 the number of shells and 64 is the number of element-pair combinations $(8 \times 8)$ in each shell. The more

123 detailed information on the structure-based features used here can be found in our previous study [14].

\section{Sequence feature generation: peptide encoding:}

125 Before inputting to the neural network, the raw peptide sequences were encoded into the numerical

126 format. Although, there are several ways in which a peptide sequence can be represented, here we tested

127 the three most popular methods of representing peptide sequence in a deep learning model. The first

128 one is a one-hot encoding, in which each peptide residue is represented as a one-hot encoded vector of

129 dimension 20 (standard amino acids) with the value corresponding to the residue was set to 1 and the

130 remaining 19 values set to 0 (Figure 1a). Secondly, in the BLOSUM62 encoding, each residue in a peptide

131 is encoded as a 20-dimensional vector from the BLOSUM62 matrix[24] thus, generating a $9 \times 20$ matrix

132 representation for the peptide (Figure $1 b$ ). For embedding, the amino acid sequence is converted into an

133 embedding vector by assigning discrete numerical codes to the residues in the peptide (Figure 1c). Each

134 of these discrete numerical variables in the embedding vector is then mapped to a vector of continuous

135 numbers (of dimension 20) thus, resulting in the embedding matrix. Unlike one-hot encoding or

136 BLOSUM62 encoding, the mapping in the embedding matrix can be improved by learning using a neural

137 network on a supervised task. For CNN based neural networks, all these encodings were mapped as 20-

138 channel 1-row 2D tensors similar to DeepMHC[10] whereas, for LSTM based neural networks, the

139 sequence information was supplied as 1-channel 2D tensor with 9 rows and 20 columns. 
a)

\begin{tabular}{|c|c|c|c|c|c|c|c|c|c|c|c|c|c|c|c|c|c|c|c|c|}
\hline & & 0 & & & & & & 0 & 0 & 10 & & & & & & & & & & \\
\hline & 0 & 0 & & & & & 0 & $c$ & $c$ & 10 & 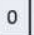 & 0 & 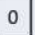 & 0 & 0 & 0 & 0 & 0 & & 0 \\
\hline & 0 & 10 & & & & & 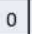 & 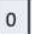 & 0 & 10 & 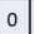 & 0 & 0 & 0 & 0 & 0 & 0 & 1 & & \\
\hline & 0 & 10 & & & & & 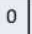 & . & 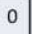 & . & 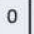 & 0 & 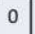 & 0 & 1 & 0 & 0 & 0 & & \\
\hline & 0 & 10 & & & & & 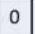 & 0 & & & & 0 & 0 & 0 & 0 & 0 & 0 & 0 & & \\
\hline & 0 & 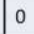 & 0 & 0 & & 0 & 0 & 0 & 0 & 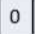 & 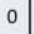 & 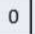 & 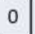 & 0 & 0 & 1 & 0 & 10 & & \\
\hline & 0 & 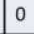 & 0 & v & 0 & 0 & 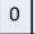 & T & 0 & 0 & 0 & 0 & 0 & 0 & 0 & 0 & 1 & 0 & & \\
\hline & 0 & 0 & 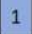 & 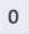 & 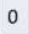 & 0 & 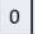 & 0 & 0 & 0 & 0 & 0 & 0 & 0 & 0 & 0 & 0 & 0 & & \\
\hline & & & & & & & T & 0 & & 0 & 0 & 0 & 0 & 0 & 0 & 0 & 0 & 0 & 0 & \\
\hline
\end{tabular}

b)

\begin{tabular}{|c|c|c|c|c|c|c|c|c|c|c|c|c|c|c|c|c|c|c|c|c|}
\hline 0 & 4 & & & & & & & & & & & & & & & & 0 & -3 & & \\
\hline 1 & & & & & & & & & & & & & & & 4 & $\rightarrow$ & -2 & 1 & & \\
\hline 2 & & & & & & & & & & & & & & & -2 & -2 & 0 & -3 & & \\
\hline 3 & -1 & & & & & & & & & & & & & $\mid-3$ & -2 & $-1 \mid$ & \begin{tabular}{|l|} 
\\
\end{tabular} & -3 & & \\
\hline 4 & -2 & & & & & & & & & & 1 & -3 & 0 & 6 & -4 & -2 & -2 & 1 & & \\
\hline 5 & 1 & & & 0 & & & 0 & 0 & -1 & -2 & -2 & 0 & $|-1|$ & -2 & $\mid-1$ & 4 & 1 & -3 & -2 & \\
\hline 6 & 0 & -1 & & 1 & & & -1 & -2 & -2 & 1 & $-1 \mid$ & -1 & $\mid-1$ & -2 & -1 & 1 & 5 & -2 & & \\
\hline 7 & -2 & -2 & & t & -3 & 0 & 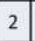 & -1 & -1 & -3 & -4 & -1 & $\mid-3$ & \begin{tabular}{|l|} 
\\
\end{tabular} & -1 & 0 & -1 & -4 & & \\
\hline & & & & & & & & & & & & & & & & & & & & \\
\hline
\end{tabular}

c) $\quad A: 1, C: 2, D: 3, E: 4, F: 5 \ldots V: 18, W: 19, Y: 20$

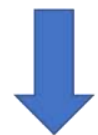

\begin{tabular}{|l|l|l|l|l|l|l|l|l|}
\hline 1 & 5 & 18 & 15 & 5 & 16 & 17 & 3 & 9 \\
\hline
\end{tabular}

Embedding Vector

Figure 1: Illustration of encoding of peptide AFVRFSTDK with three different encoding techniques used in this study a) In one-hot encoding, each position is represented as a 20-dimensional vector with the value of corresponding residue kept as 1 and remaining 19 residues as $0 \mathrm{~b}$ ) In BLOSUM62 encoding, each position is represented by the vector corresponding to the residue from the BLOSUM62 matrix c) In embedding vector, each residue is assigned an integer value and the peptide is represented as embedding vector formed out of those integer values.

Model Architecture:

148 The models used in this study consist of two modules: the structure module and the sequence module.

149 The structure module consists of three convolution layers with each convolution layer is followed by a

150 max-pooling layer. Batch-normalization and dropout layers are added after each convolution layer with

151 "relu" as the activation function. The organization of the structural module was mainly inspired by our

152 previous work, OnionNet[14]. For the sequence module, we tested both the convolution-based neural

153 network and the LSTM-based neural network. The LSTM-based neural network in sequence module

154 comprises of two LSTM-layers with each LSTM layer is followed by batch normalization and dropout layer

155 (Figure 2a). The activation function used with LSTM layers is "tanh". The convolution-based neural

156 network in sequence module comprises of three convolution layers with each convolution layer is 
157 followed by a max-pooling layer (Figure 2b). The outputs of the structure and sequence blocks are

158 received by the individual fully connected (FC) layer of 1024 neurons. The outputs are combined and fed

159 into another FC layer of 512 neurons to predict the binding affinity. Deep learning models generally have

160 millions of trainable parameters which is often larger than the number of training samples. This is because

161 the input features often follow a very complicated relationship with target values and a network with few

162 trainable parameters will not be able to represent the desired mapping. Having many parameters would

163 make the network more flexible so that the features can be mapped properly to the target values. To

164 reduce generalization error and prevent overfitting, we not only used implicit regularizations (e.g.

165 dropout) but also employ explicit regularization L2 in our model with $\lambda=0.001$ [25-27].

166 We tested the three different peptide sequence encoding methods along with these two model

167 architectures in the sequence module. The three sequence encoding methods have also been used to

168 predict the binding affinity of peptides with MHC: one-hot encoding[10], BLOSUM62 encoding[28], and

169 embedding vector representation embedding[6,29]. Thus, we tested six models that differ in terms of

170 peptide sequence encoding and the architecture of the sequence module. Since, the input features and

171 the architecture of the structure module are the same in all the models, therefore, the tested models are

172 named based on their peptide sequence encoding and network architecture used in the sequence module

173 (Table 1).

\begin{tabular}{|c|c|c|c|}
\hline S/No. & Model Name & Peptide encoding & Sequence Module Type \\
\hline 1 & bls_cnn & BLOSUM62 encoding & CNN based \\
\hline 2 & onehot_cnn & One-hot encoding & CNN based \\
\hline 3 & emb_cnn & Embedding vector encoding & CNN based \\
\hline 4 & bls_Istm (OnionMHC) & BLOSUM62 encoding & LSTM based \\
\hline 5 & onehot_Istm & One-hot encoding & LSTM based \\
\hline 6 & emb_Istm & Embedding vector encoding & LSTM based \\
\hline
\end{tabular}

Table 1: Models with different peptide sequence encoding and the neural network architecture used in the sequence module. Three ways of peptide sequence encoding (one-hot, blosum62, embedding vector) combined with convolutional neural network 
a)

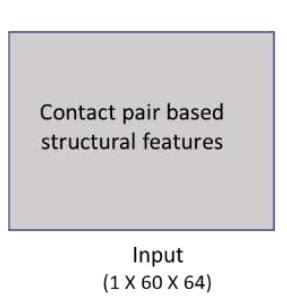

$(1 \times 60 \times 64)$
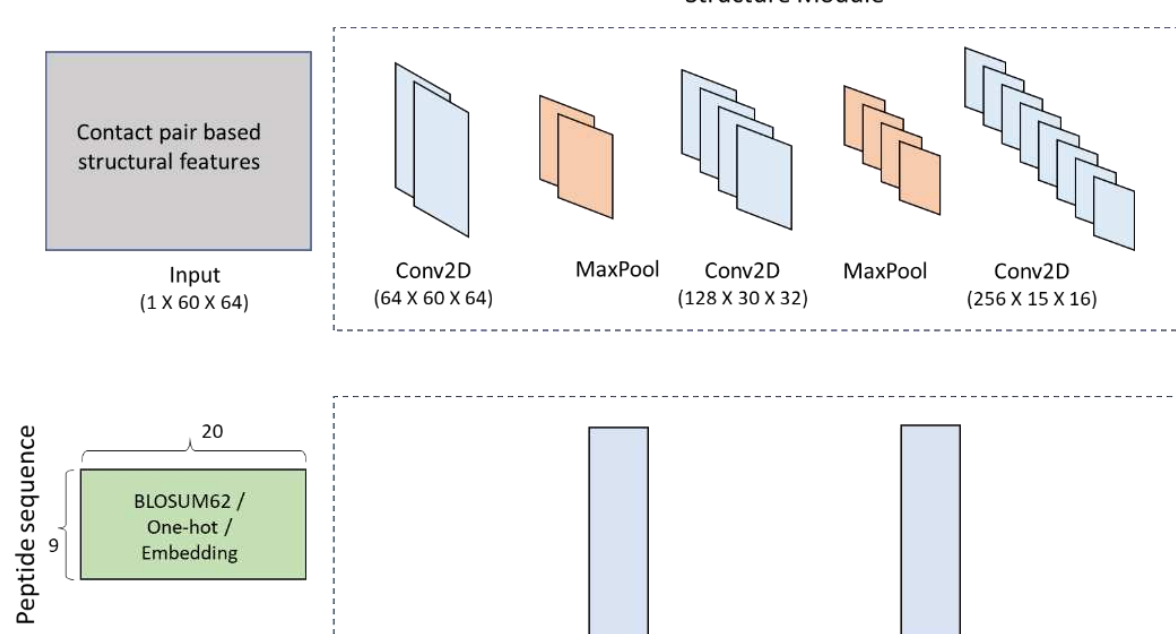

Input Matrix $(9 \times 20)$

b)
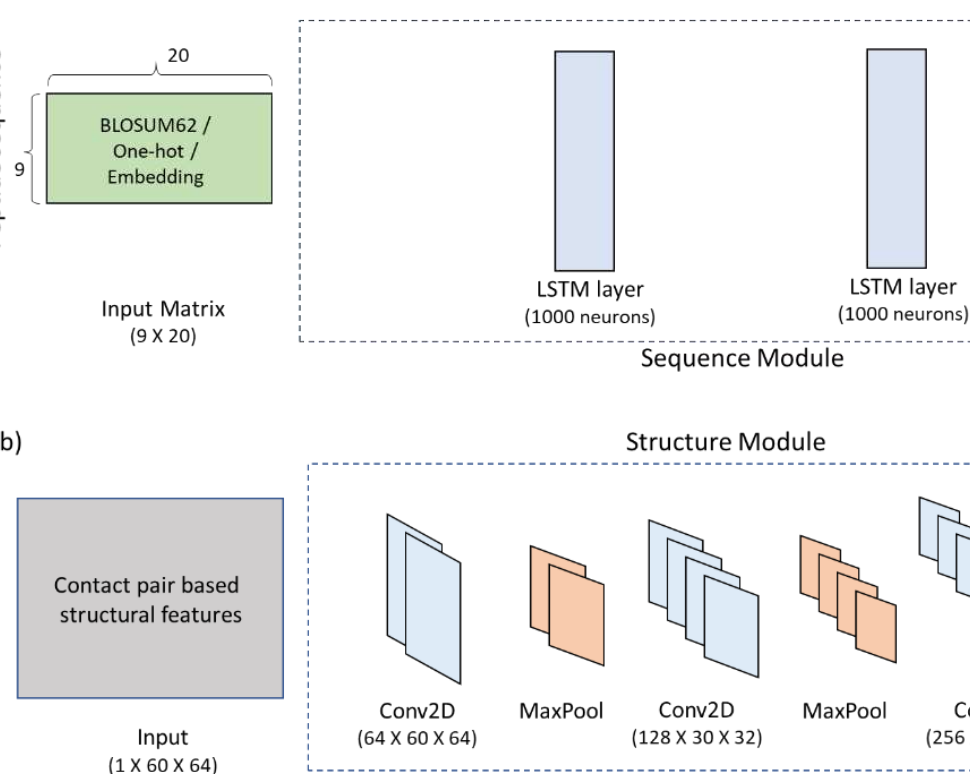

Sequence Module

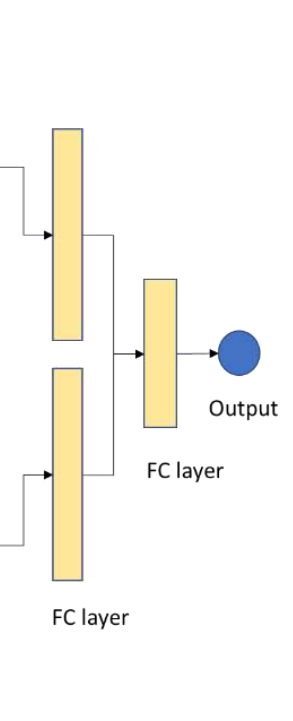

Structure Module
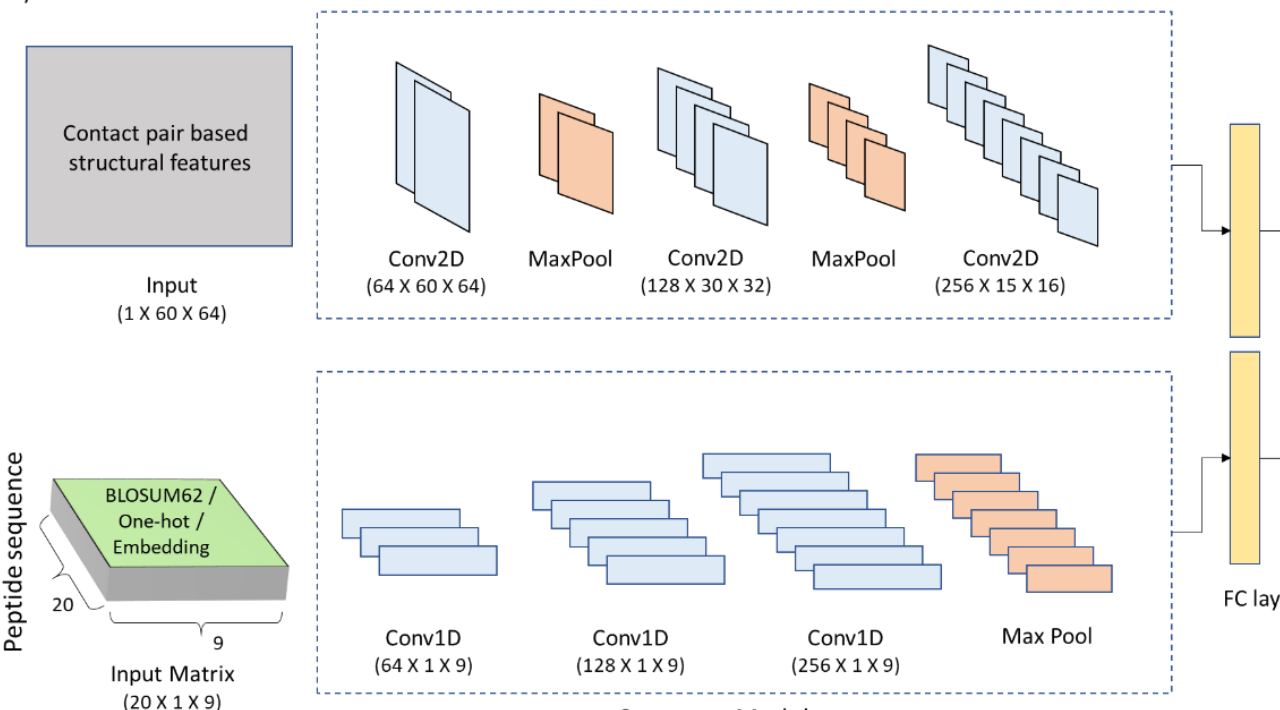

20 channel 1 -row $2 \mathrm{D}$ tensor
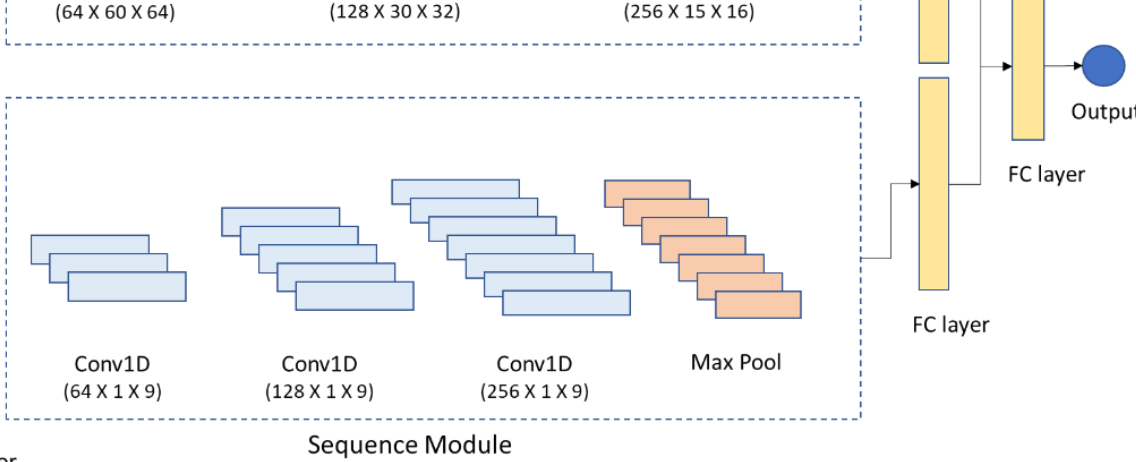

FC layer

Sequence Module

Figure 2: Architectures tested in this study with structure module based on Convolutional Neural Network: a) Long Short-Term

Memory based neural network was used in sequence module with two Istm layers of 1000 neurons each and its output is combined with that of structure module using Fully Connected (FC) layer b) Convolutional Neural Network-based neural network was used in sequence module with three 1-D convolutional layers (of filter size 64, 128, 256), followed by maximum pooling and its output is also combined with that of structure module using Fully Connected (FC) layer 
188 The binding affinities (in $\mathrm{nM}$ ) in the collected dataset were transformed to numbers within 0 to 1 with a 189 cap of $50,000 \mathrm{nM}$ :

$$
\text { affintiy }_{\text {transformed }}=\min \left(1-\frac{\log (\text { affinity })}{\log (50000)}, 1\right)
$$

190 as previously described in $[5,11,28]$.

191 The hyperparameters involving the number of neurons, number of layers, and batch size were

192 optimized using the five-fold cross-validation (Figure S1, Additional File 1). The model with optimized

193 hyperparameters was trained with mean-squared-error used as the loss function.

$$
\text { Loss }=\frac{1}{n} \sum_{i}^{n}\left(\widetilde{y}_{i}-y_{i}\right)^{2}
$$

194 The dataset was randomly divided into 5-folds and the model was trained for 600 epochs with 195 earlystopper of 30 epochs if the validation loss did not decrease more than 0.0001 . The framework used

196 for the model implementation was keras-tensorflow2.0 on NVIDIA-GeForce GTX 1080 Ti.

197 For the cross-validation, an ensemble of 5 deep neural networks was generated for each model by training 198 the network on 4 folds and testing it on the remaining fold. For the final prediction on the benchmark 199 dataset, two more trials of each of the 5 cross-validated neural networks were performed thus, resulting 200 in 15 deep neural networks for each model. The average prediction score of all these 15 networks was 201 taken as the final prediction score for a peptide. This methodology was inspired by the recently published 202 model ACME[11].

203

204 Performance Metric: 
205 The performance of the model was independently evaluated on the benchmark data obtained from IEDB

206 and compared with the existing models such as NetMHCpan4.0, convMHC, HLAthena. The performance

207 metrics used for comparison is the Area Under Receiver Operating Curve (AUC) and the Spearman Rank

208 Correlation Coefficient (SRCC). This SRCC metric is the same as adopted by the weekly benchmark dataset

209 provided by IEDB (http://tools.iedb.org/auto bench/mhci/help). In the weekly benchmark dataset, the

210 performance was reported in terms of SRCC even for binary data also. In that case, the SRCC calculation

211 was done by keeping the target values as either 0 (non-binder) or 1 (binder) which is different from ic50

212 or half-life measurements.

\section{Molecular Dynamics Simulation}

214 The stability of the modeled complex structures was explored by performing molecular dynamics (MD)

215 simulations using GROMACS[23]. The bonding and non-bonding parameters were derived from

216 AMBER99SB-ILDN force field[30]. The simulation was performed in a cubic box with water molecules and

217 ions $\left(\mathrm{Na}^{+}\right.$and $\left.\mathrm{Cl}^{-}\right)$concentration of $0.15 \mathrm{M}$ at $300 \mathrm{~K}$. The system was equilibrated before carrying out the

218 production simulation. Under NVT conditions, the equilibration was performed for $100 \mathrm{ps,} \mathrm{then} \mathrm{under}$

219 NPT conditions for 100 ps using 2 fs time step, Berendsen thermostat $(\tau=0.1$ ps), and barostat $(\tau=2$ ps).

220 The bonds and angles were constrained by the LINCS algorithm[31]. The production simulation was

221 carried out under NPT conditions for 50 ns with repeats. A leap-frog algorithm[32] was used to integrate

222 Newton's equation of motion. The cut-offs for both van der Waals (VDW) interaction and short-range

223 electrostatic interaction were set to $1.0 \mathrm{~nm}$, and the particle mesh Ewald (PME) method[33] was

224 employed to deal with the long-range electrostatic interactions.

\section{Cell culture}


226 T2 cells were cultured in Roswell Park Memorial Institute (RPMI) 1640 media (Hyclone) supplemented

227 with 20\% heat-inactivated Fetal Bovine Serum (FBS; Hyclone) and 1\% penicillin/streptomycin (Hyclone) at

$22837^{\circ} \mathrm{C}$ in a $\mathrm{CO}_{2}$ incubator.

\section{Peptide binding assay}

230 Peptides, as shown in Table 5 were synthesized from Peptide 2.0, USA, in a lyophilized form with $>95 \%$

231 purity. The peptides were dissolved in either sterile water or 10\% DMSO depending on the sequences.

232 The binding of the candidate peptides to the HLA-A*02:01 molecules was assessed by measuring the

233 induction of surface expression of HLA-A*02:01 molecules on peptide-pulsed T2 cells following an

234 established protocol[34] with a few modifications. The T2 cells were seeded in a 24 well plate and then

235 treated with the peptides at $100 \mu \mathrm{M}$ concentration. The peptide-pulsed $\mathrm{T} 2$ cells were then incubated at

$23637^{\circ} \mathrm{C}$ in a $\mathrm{CO}_{2}$ incubator for 12 to $16 \mathrm{~h}$. The cells were then stained with HLA-A02:01 specific mAb

237 conjugated to FITC for $30 \mathrm{~min}$ at room temperature and then analyzed by flow cytometer. The

238 fluorescence index (FI) was calculated using the following formula:

$$
\mathrm{FI}=\frac{\left[\text { mean fluorescence intensity }(\mathrm{MFI})_{\text {sample }}-\mathrm{MFI}_{\text {background }}\right]}{\mathrm{MFI}_{\text {background }}}
$$

240 where $\mathrm{MFI}_{\text {background }}$ represents the value without peptide. $\mathrm{FI}>1.5$ indicated that the peptide had a high

241 affinity for HLA-A*02:01 molecules, $1.0<\mathrm{FI}<1.5$ indicated that the peptide had a moderate affinity for 242 the HLA-A*02:01 molecules, and $0.5<\mathrm{FI}<1.0$ indicated that the peptide had low affinity for the 243 HLA-A*02:01 molecules.

\section{Results and Discussions}


Five-fold Cross-Validation:

247 We evaluated the performance of all the six models using the five-fold cross-validation. The entire data

248 was divided into five-folds and 5 different models were generated with each model tested on one of the

2495 folds and trained on the remaining 4 folds. The performance evaluation of the above-mentioned models

250 (Table 1) was done in terms of both AUC and SRCC. Overall, the LSTM-based neural network in the

251 sequence module achieved better performance with our structure module compared to the CNN-based

252 neural network in the sequence module as shown in Table 2 (Table S1, Additional File 2). The peptide

253 encoding does not have much difference in the model performance with the LSTM-based neural network

254 in the sequence module. However, for the sequence module with a CNN-based neural network, the one-

255 hot encoding performed a bit worse than the blosum62 encoding or the embedding vector

256 representation.

\begin{tabular}{|l|c|c|c|c|c|c|}
\hline & emb_Istm & onehot_Istm & bls_Istm & emb_cnn & onehot_cnn & bls_cnn \\
\hline AUC & 0.955 & 0.953 & 0.954 & 0.943 & 0.878 & 0.941 \\
\hline SRCC & 0.816 & 0.816 & 0.821 & 0.798 & 0.677 & 0.799 \\
\hline
\end{tabular}

Table 2: Summary of five-fold cross-validation measured in terms of area under the receiver operating curve (AUC) and Spearman Rank Correlation Coefficient (SRCC)

260 Independent Evaluation:

261 For independent evaluation, we downloaded the weekly benchmark dataset (since 21/03/2014) from

262 IEDB (http://tools.iedb.org/auto bench/mhci/weekly/) for all the 9-mer peptides belonging to HLA-

263 A*02:01 receptors. The performance of all models was evaluated on the weekly benchmark dataset in

264 terms of AUC and SRCC (Figure 3, 4). Among all the models generated, bls_Istm (OnionMHC) achieved the

265 best performance both in terms of AUC and SRCC on the IEDB benchmark dataset (Figure 3). The 
266 performance of OnionMHC was compared with other different algorithms (Figure 4) which were already 267 evaluated in previous work [35]. We also compared the performance of our model with the current state268 of-the-art baseline model NetMHCpan4.0 (http://www.cbs.dtu.dk/services/NetMHCpan/) and the 269 convolution-based neural network model convMHC. The standalone version of NetMHCpan4.0 was 270 downloaded from http://www.cbs.dtu.dk/services/NetMHCpan/ and installed as instructed. The 271 predictions of the extracted benchmark dataset were made and AUC and SRCC were calculated.

272 NetMHCpan4 gives two types of outputs which are the binding probability of peptide with HLA receptor 273 (NetMHCpan4-L) and the binding affinity of the peptide with the receptor (NetMHCpan4-B). For 274 convMHC, the predictions of the extracted benchmark dataset were made from the webserver 275 (http://jumong.kaist.ac.kr:8080/convmhc). We also compared our model with the recently published 276 HLAthena[15] which can be accessed from the webserver (http://hlathena.tools/ ). On average, our 277 model achieved a better performance both in terms of AUC and SRCC compared to NetMHCpan4-L, 278 convMHC, and HLAthena and the performance was comparable to NetMHCpan4-B (Table 3).

279 The IEDB benchmark dataset consists of data of three measurement types: binary, ic50, and $\mathrm{t} 1 / 2$. In one 280 of the references, we found some discrepancies with the peptides identified as positives. The reference 281 id 1026840 showed the same peptides with two different measurements: ic50 and t1/2. With the 282 conventional cut-offs of ic50 $(<500 \mathrm{nM})$ and $\mathrm{t} 1 / 2(>120 \mathrm{~min})$ for a peptide to be counted as positive, the 283 numbers of binders were different (positives) in both measurements. There were 14 positives out of 24 284 peptides according to ic50 cut-off and 7 positives out of 24 according to $t 1 / 2$ measurement. Because of 285 this discrepancy, we did not consider this reference id for evaluating our model. 


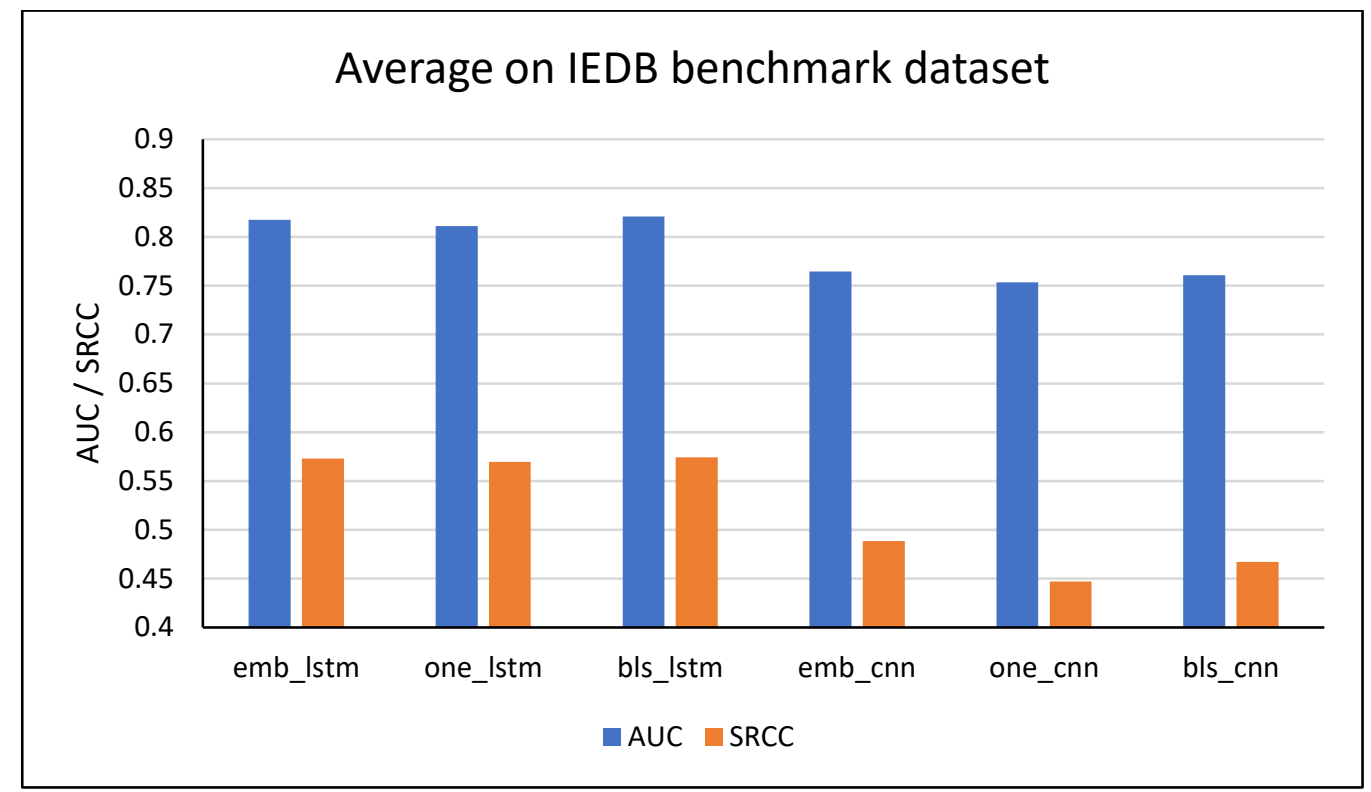

Figure 3: Performance evaluation of the six generated models on the IEDB benchmark dataset

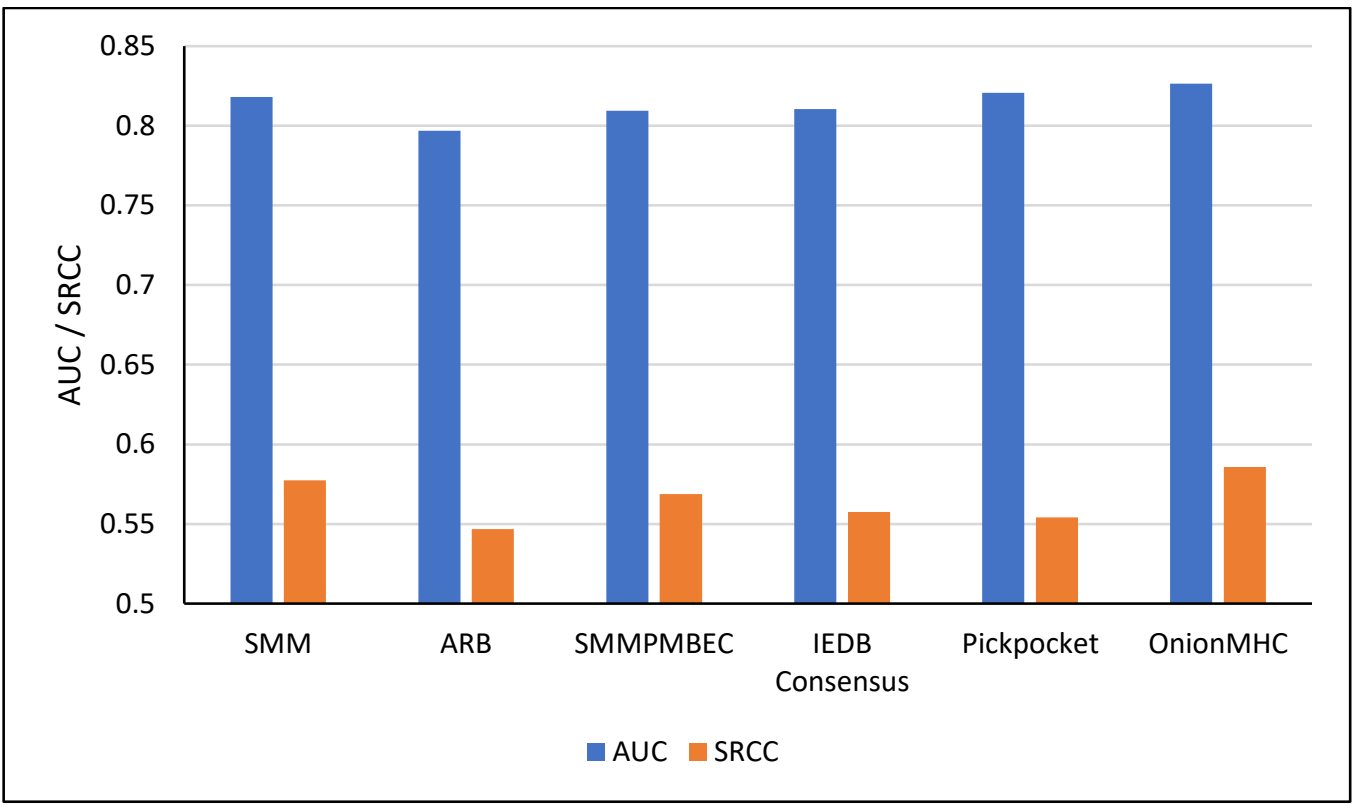

Figure 4: Performance comparison of OnionMHC with different prediction algorithms (Trolle et. al 2015) on the most recent IEDB benchmark dataset, measured in terms of Spearman Rank Correlation Coefficient (SRCC) and area under receiver operating curve (AUC) 


\begin{tabular}{|c|c|c|c|c|c|c|c|c|c|c|c|c|c|}
\hline \multirow{2}{*}{ IEDB Ref. } & \multirow{2}{*}{$\begin{array}{c}\text { Peptide } \\
\text { Count }\end{array}$} & \multirow{2}{*}{$\begin{array}{c}\text { Positive } \\
\text { Count }\end{array}$} & \multirow{2}{*}{$\begin{array}{c}\text { Measu } \\
\text { rement } \\
\text { type }\end{array}$} & \multicolumn{2}{|c|}{ NetMHCpan4 - L } & \multicolumn{2}{|c|}{ NetMHCpan4 - B } & \multicolumn{2}{|c|}{ convMHC } & \multicolumn{2}{|c|}{ HLAthena } & \multicolumn{2}{|c|}{ OnionMHC } \\
\hline & & & & AUC & SRCC & AUC & SRCC & AUC & SRCC & AUC & SRCC & AUC & SRCC \\
\hline 1033576 & 191 & 13 & binary & 0.619 & 0.104 & 0.63 & 0.113 & 0.63 & 0.09 & 0.596 & 0.084 & 0.666 & 0.145 \\
\hline 1029824 & 77 & 56 & binary & 0.479 & -0.033 & 0.556 & 0.087 & 0.588 & 0.14 & 0.526 & 0.039 & 0.565 & 0.101 \\
\hline 1028928 & 13 & 11 & binary & 0.955 & 0.57 & 0.955 & 0.57 & 0.932 & 0.542 & 0.955 & 0.57 & 0.955 & 0.57 \\
\hline 1027588 & 19 & 8 & binary & 0.829 & 0.564 & 0.841 & 0.584 & 0.705 & 0.35 & 0.875 & 0.642 & 0.773 & 0.467 \\
\hline 1027471 & 45 & 6 & binary & 0.859 & 0.423 & 0.838 & 0.398 & 0.823 & 0.381 & 0.889 & 0.458 & 0.774 & 0.322 \\
\hline 1027079 & 18 & 13 & binary & 0.846 & 0.538 & 0.846 & 0.538 & 0.815 & 0.49 & 0.815 & 0.49 & 0.769 & 0.418 \\
\hline 1026840 & 357 & 76 & binary & 0.877 & 0.534 & 0.904 & 0.573 & 0.872 & 0.529 & 0.819 & 0.452 & 0.886 & 0.547 \\
\hline 1026941 & 10 & 6 & binary & 1 & 0.853 & 0.917 & 0.711 & 1 & 0.853 & 0.875 & 0.64 & 1 & 0.853 \\
\hline 1031894 & 434 & 368 & ic50 & 0.776 & 0.531 & 0.901 & 0.743 & 0.82 & 0.603 & 0.743 & 0.479 & 0.932 & 0.8 \\
\hline 1031072 & 28 & 23 & ic50 & 0.974 & 0.739 & 1 & 0.875 & 0.957 & 0.772 & 0.913 & 0.727 & 0.974 & 0.856 \\
\hline 1033071 & 113 & 41 & ic50 & 0.866 & 0.77 & 0.876 & 0.812 & 0.851 & 0.759 & 0.871 & 0.787 & 0.872 & 0.819 \\
\hline 1028790 & 55 & 47 & ic50 & 0.598 & 0.628 & 0.582 & 0.626 & 0.612 & 0.607 & 0.516 & 0.508 & 0.588 & 0.643 \\
\hline 1028553 & 22 & 2 & ic50 & 0.7 & 0.432 & 0.85 & 0.574 & 0.85 & 0.603 & 0.575 & 0.229 & 0.925 & 0.568 \\
\hline 1028554 & 44 & 7 & ic50 & 0.838 & 0.539 & 0.876 & 0.721 & 0.753 & 0.417 & 0.9 & 0.641 & 0.884 & 0.679 \\
\hline 1024516 & 51 & 45 & ic50 & 0.663 & 0.685 & 0.63 & 0.667 & 0.693 & 0.658 & 0.556 & 0.558 & 0.678 & 0.728 \\
\hline 1026941 & 10 & 6 & ic50 & 1 & 0.864 & 0.917 & 0.717 & 1 & 0.422 & 0.875 & 0.724 & 1 & 0.824 \\
\hline 1026371 & 85 & 49 & $\mathrm{t} 12$ & 0.781 & 0.564 & 0.811 & 0.566 & 0.784 & 0.517 & 0.793 & 0.55 & 0.803 & 0.543 \\
\hline 1028285 & 881 & 549 & $\mathrm{t} 12$ & 0.822 & 0.656 & 0.865 & 0.721 & 0.829 & 0.658 & 0.799 & 0.626 & 0.831 & 0.66 \\
\hline \multicolumn{4}{|c|}{ Average } & 0.805 & 0.553 & 0.822 & 0.588 & 0.806 & 0.521 & 0.772 & 0.511 & 0.826 & 0.586 \\
\hline
\end{tabular}

297 Table 3: AUC Performance evaluation of OnionMHC with NetMHCpan and ConvMHC on IEDB benchmark dataset for HLA-

A*02:01 with peptide length 9

300 All these models (OnionMHC, NetMHCpan4, HLAthena, and convMHC) utilized the data from IEDB

301 (http://tools.iedb.org/main/datasets/) in their training set and their evaluation was done on the

302 benchmark data also provided by IEDB under three measurement values (binary, ic50, and t1/2).

303 However, there is an overlap between these two datasets, so we removed the overlapping peptides for 
304 the three measurement values (Figure 5) and re-calculated the spearman correlation (SRCC) and area 305 under the receiver operating curve (AUC) on the benchmark peptides. These benchmark peptides have 306 not been seen by OnionMHC during the training or the validation. Among the datasets with three 307 measurement values, OnionMHC performed notably better than convMHC, NetMHCpan4-L, and 308 HLAthena on ic50 measurement values, and equally well with NetMHCpan4-B (Table 4, Figure 6). For the 309 data with the binary form, OnionMHC still performed better than HLAthena and similarly well with 310 convMHC, NetMHCpan4-L, and NetMHCpan4-B. In the half-time measurement dataset, we found that 311 the peptide TVYDINNEV had two different measurement values of $120 \mathrm{~min}$ and $170.4 \mathrm{~min}$. With the 312 conventional cut-off of $>120$ min for classifying a peptide as a binder, this peptide would have created a 313 discrepancy, so we removed it from the analysis. For the half-time measurement dataset, OnionMHC did 314 not perform as well as other models however, the performance was still comparable. 
a)

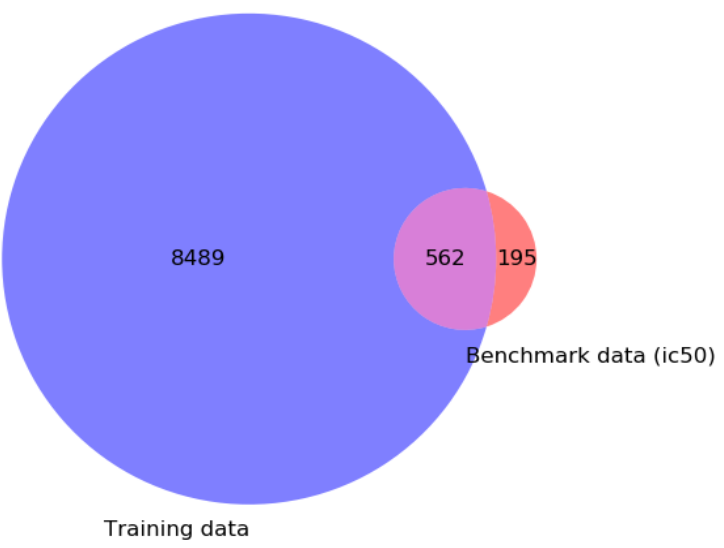

b)

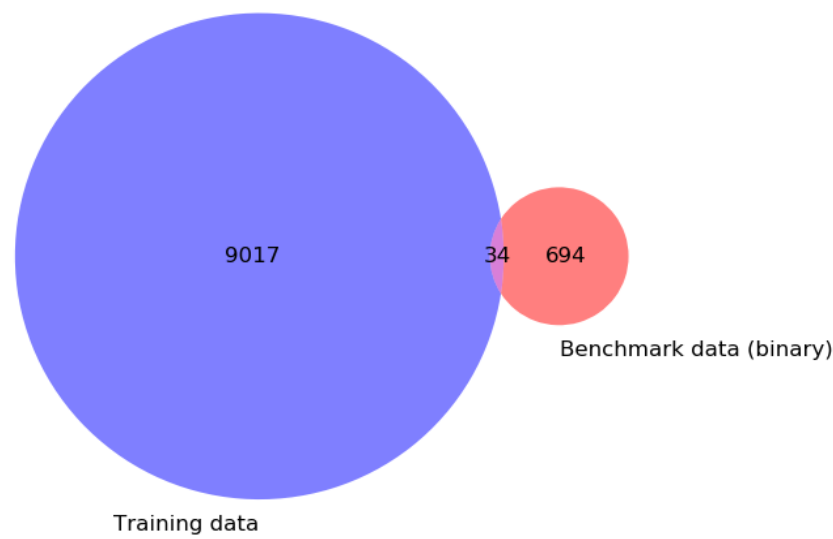

c)

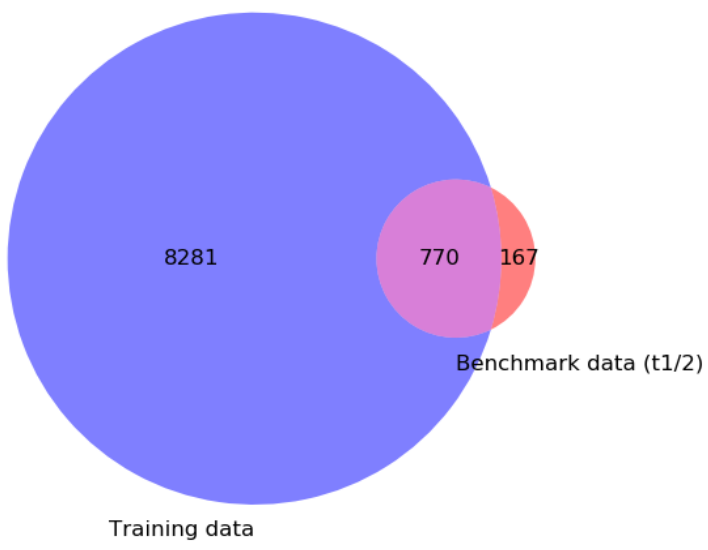

Figure 5: Overlapping peptides between the training data and benchmark data with measurement value: a) binary; b) ic50; c) half-life (t1/2)

\begin{tabular}{|c|c|c|c|c|c|}
\hline & OnionMHC & NetMHCpan4.0-L & NetMHCpan4.0-B & convMHC & $\begin{array}{c}\text { Hlathena } \\
\text { predictor }\end{array}$ \\
\hline $\begin{array}{c}\text { For ic50 only } \\
\text { (195 peptides) }\end{array}$ & & & & & \\
\hline SRCC & 0.790 & 0.716 & 0.787 & 0.697 & 0.741 \\
\hline AUC & 0.885 & 0.857 & 0.882 & 0.834 & 0.869 \\
\hline $\begin{array}{c}\text { For binary only } \\
\text { (694 peptides) }\end{array}$ & & & & & \\
\hline SRCC & 0.529 & 0.524 & 0.539 & 0.519 & 0.477 \\
\hline AUC & 0.860 & 0.856 & 0.866 & 0.852 & 0.824 \\
\hline
\end{tabular}




\begin{tabular}{|c|c|c|c|c|c|}
\hline $\begin{array}{c}\text { For half-life only } \\
\text { (167 peptides) }\end{array}$ & & & & & \\
\hline SRCC & 0.465 & 0.618 & 0.603 & 0.532 & 0.611 \\
\hline AUC & 0.754 & 0.789 & 0.819 & 0.790 & 0.761 \\
\hline
\end{tabular}

Table 4: Evaluation of models on the benchmark data after removing the overlapping peptides
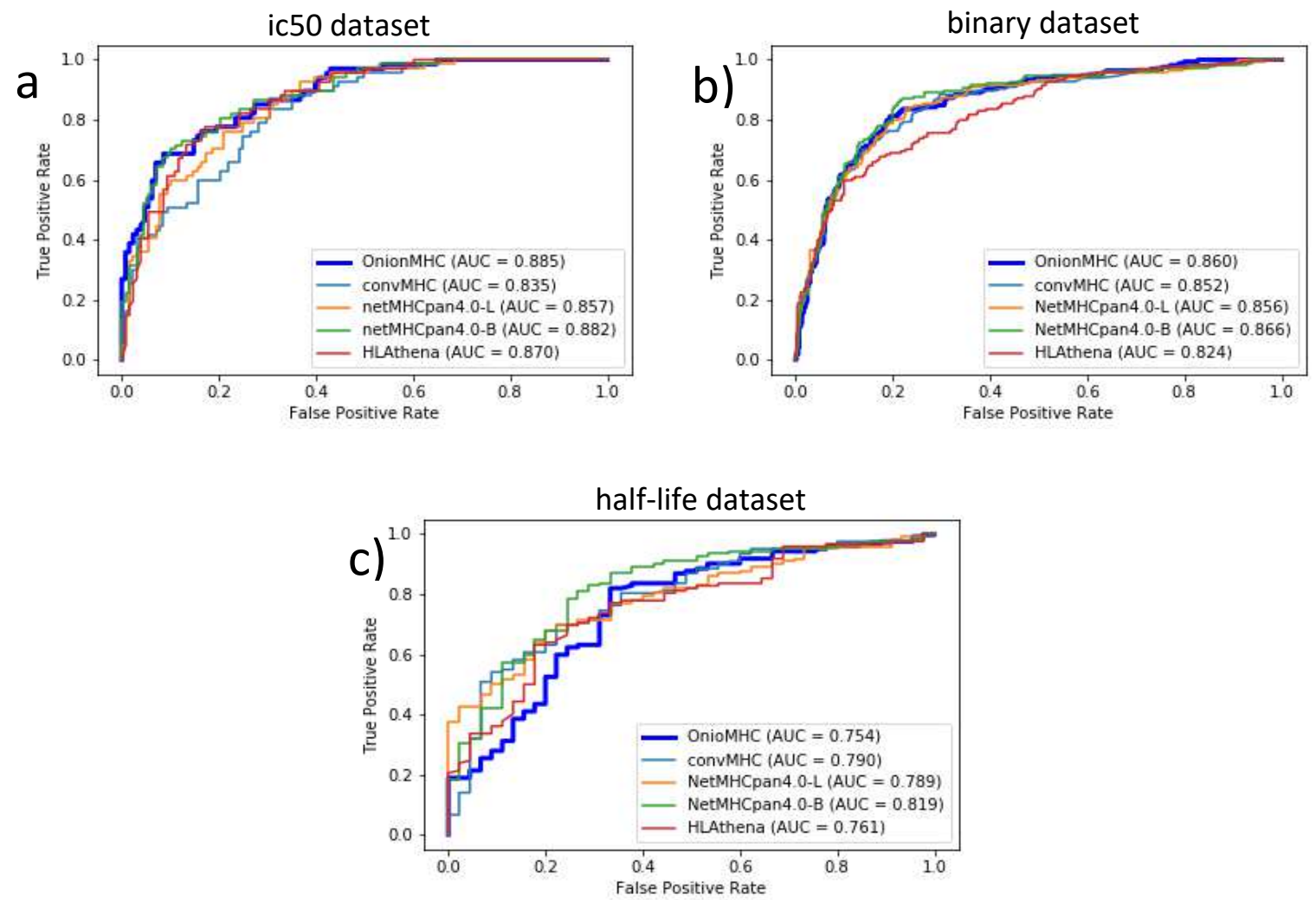

Figure 6: Area under the receiver operating curves for the non-overlapping peptides a) ic50 dataset (195 peptides) b) binary dataset (694 peptides) c) half-life dataset (167 peptides)

\section{Model Ablation}

324 OnionMHC has both structure as well as sequence modules. To understand the importance of each

325 module in predicting the binding affinity of a peptide, we performed model ablation analysis on our

326 model. In this analysis, either of the modules is removed and the rest of the model is trained similarly to

327 the original model and evaluated for its performance on the IEDB benchmark dataset. As shown in Figure

3287 , the sequence module is the main contributor towards the binding affinity prediction, however, the 
329 addition of the structure module further improves the predictive power of the sequence module. The

330 structure information was obtained mostly from modeling instead of experimentally determined PDB

331 structures. The inaccuracy or the noise in the structural information may damage the quality of the

332 structural models. This could be the reason why the sequence module performs better than the structure

333 module in the prediction of binding affinity.

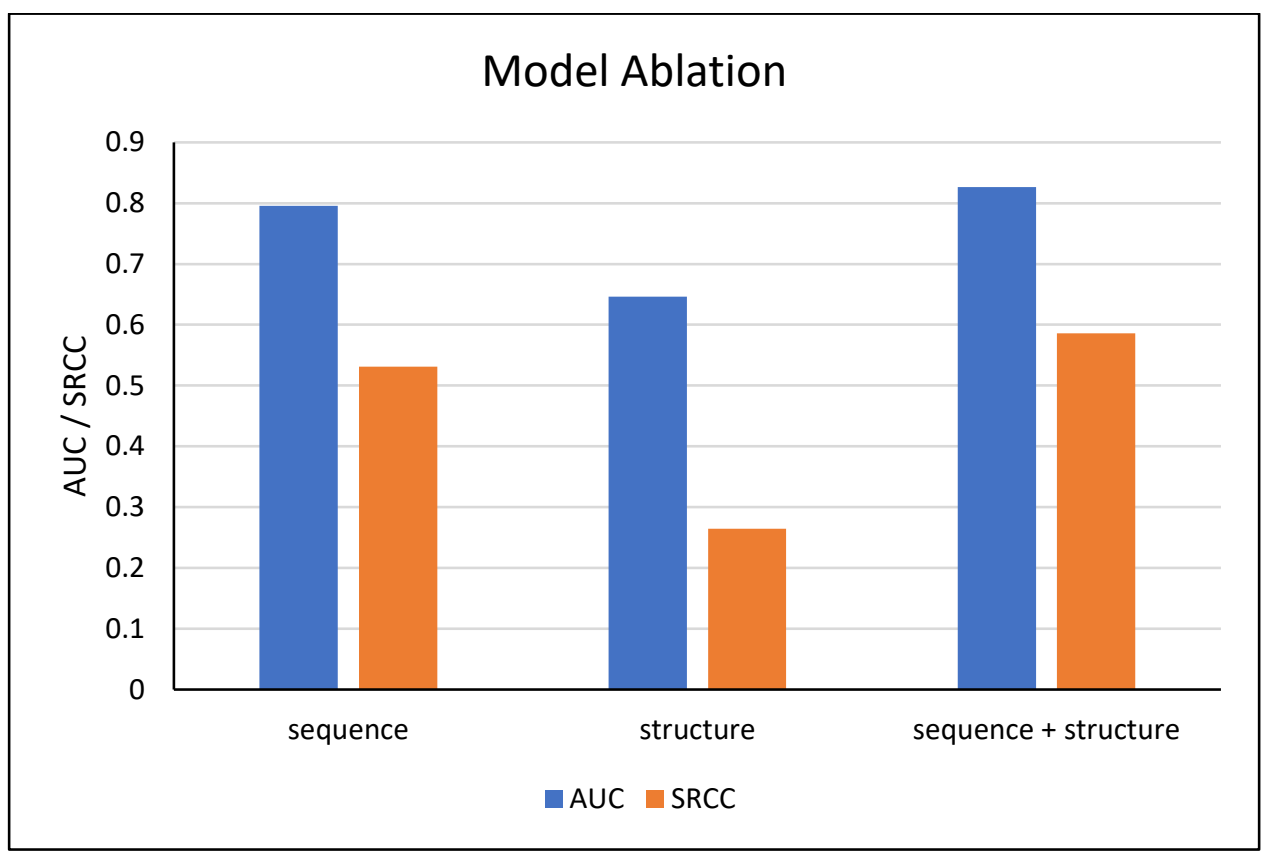

Figure 7: Model Ablation analysis of OnionMHC showing the individual performance of sequence and structure module on IEDB benchmark dataset

\section{Demonstration by Molecular Dynamics Simulations}

338 To demonstrate the stability of the predicted binding peptides as well as the non-binding peptides in the

339 complex with the receptor, we performed molecular dynamics simulations on the receptor-peptide

340 system. These peptides in the IEDB benchmark data have already been validated experimentally[35]. In

341 total we randomly selected 6 peptides, 3 of which are predicted to be binders with the binding affinity

342 less than $500 \mathrm{nM}$ (FLDEFMEGV, LLIDLTSFL, NLIDFNFKL) and the remaining 3 were non-binders with the

343 binding affinity greater than $500 \mathrm{nM}$ (ANRAVKPTG, DIKVQFQSG, TPTAYRPPN). We calculated the residue-

344 wise root mean square fluctuation (RMSF) of the peptide backbone (Figure 8). The peptide backbone, 
especially those at the termini, shows larger RMSF for the non-binding peptides compared to that of the

346 binding peptides. The peptide binds to class I MHC primarily through the invariant peptidic termini[36,

347 37]. The second residue and the last residue (residue 9) of the peptide play an important role in its binding

348 affinity to the HLA-A*02:01 receptors [38-40]. Higher RMSF of peptide backbone at the amino terminus

349 indicates that the peptide is highly flexible at the termini thus, loosely anchored to the receptor. The low

350 RMSF for the binding peptides at the amino terminus indicates the strong binding of the peptide to the

351 HLA receptor thus, which is consistent with the predictions made by OnionMHC. A similar idea of using

352 the fluctuations from MD trajectories to distinguish the true binding from decoys has also proved to be

353 quite successful[41].

354 We also performed the binding energy calculations using g_mmpbsa [42] available at

355 https://rashmikumari.github.io/g mmpbsa/. The molecular mechanics van der Waals potential energy

356 is lower for binders than non-binders which is consistent with the predictions made by our model.

357 However, the total binding energy that includes both molecular mechanics potential energy and free

358 energy of solvation is unable to capture the same trend (Table S2, Additional File 3). Thus, the MMPBSA

359 method is unable to distinguish the binders from non-binders.

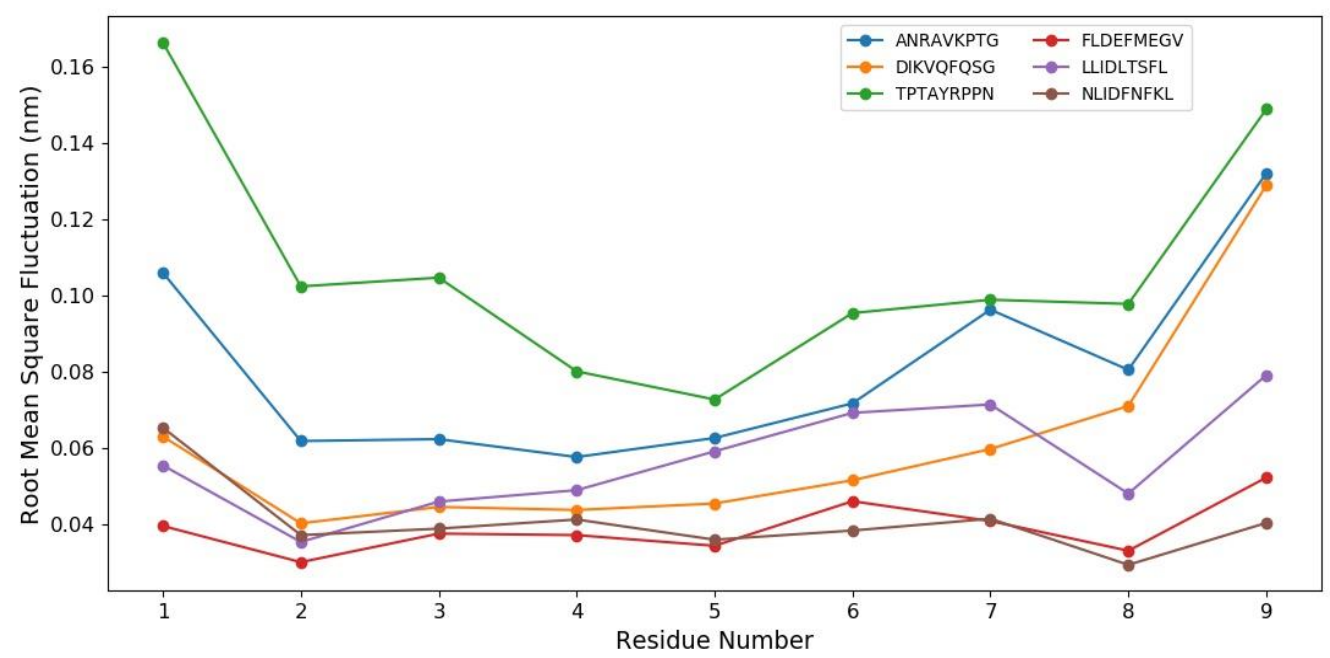

Figure 8: Root Mean Square Fluctuation ( $\mathrm{nm}$ ) of the residues in the peptide in the Molecular Dynamics simulation. 
363 We also tested our model on the peptides that were obtained from neoantigen analysis on the patient

364 exome sequencing samples from breast cancer patients. Our model OnionMHC performed equally well

365 as NetMHCpan4-L and convMHC with correctly predicting 8 out of 11 peptides (Table 5). Two peptides

366 LLAYRAYEL and NLWPVPHLL were predicted as false positives by all the models whereas RLPIFRLSI was

367 predicted as false positive by NetMHCpan4-L and convMHC only. Of the 11 peptides tested,

368 NetMHCpan4-L and convMHC predicted 3 false positives (LLAYRAYEL, NLWPVPHLL, and RLPIFRLSI),

369 NetMHCpan4-B predicted 2 false positives (LLAYRAYEL, NLWPVPHLL) whereas OnionMHC predicted 2

370 false positives (LLAYRAYEL and NLWPVPHLL) and 1 false negative (GLIEEGEKL). With 2 false positives,

371 OnionMHC and NetMHCpan4-B showed a better positive predictive value (PPV) of 0.67 as compared to

372 the value of 0.57 by NetMHCpan4-L and convMHC.

\begin{tabular}{|c|c|c|c|c|c|c|c|c|c|c|c|}
\hline \multirow{2}{*}{ S.No. } & \multirow{2}{*}{$\begin{array}{l}\text { Peptide } \\
\text { Sequence }\end{array}$} & \multicolumn{2}{|c|}{ convMHC } & \multicolumn{2}{|c|}{ NetMHCpan4.0-L } & \multicolumn{2}{|c|}{ NetMHCpan4.0-B } & \multicolumn{2}{|c|}{ OnionMHC } & \multicolumn{2}{|c|}{$\begin{array}{l}\text { Experimental } \\
\text { results }\end{array}$} \\
\hline & & Score & Binding & Score & Binding & Score & Binding & Score & Binding & $\begin{array}{l}\text { MFI } \\
\text { Score }\end{array}$ & Binding \\
\hline 1 & FLPPPECPV & 0.8989 & YES & 0.676588 & YES & 0.856453 & YES & 13.31661 & YES & 3.13 & YES \\
\hline 2 & FLLPPECPV & 0.9211 & YES & 0.799927 & YES & 0.759096 & YES & 6.828626 & YES & 2.51 & YES \\
\hline 3 & RLPIFSRLS & 0.096 & NO & 0.003027 & $\mathrm{NO}$ & 0.406621 & NO & 9656.127 & $\mathrm{NO}$ & -0.052 & NO \\
\hline 4 & RLPIFRLSI & 0.8179 & YES & 0.170836 & YES & 0.13418 & $\mathrm{NO}$ & 610.4254 & $\mathrm{NO}$ & -0.043 & NO \\
\hline 5 & LLAYRAYEL & 0.8889 & YES & 0.495571 & YES & 0.68252 & YES & 18.51016 & YES & -0.066 & NO \\
\hline 6 & LLACRAYEL & 0.8559 & YES & 0.31138 & YES & 0.750783 & YES & 40.86188 & YES & 0.63 & YES \\
\hline 7 & GLMEEGEKL & 0.8416 & YES & 0.936163 & YES & 0.68583 & YES & 175.2056 & YES & 2.33 & YES \\
\hline 8 & GLIEEGEKL & 0.8378 & YES & 0.886652 & YES & 0.593803 & YES & 682.3957 & $\mathrm{NO}$ & 0.66 & YES \\
\hline 9 & NLWPVPHLL & 0.9146 & YES & 0.970742 & YES & 0.809898 & YES & 13.40361 & YES & 0.03 & NO \\
\hline 10 & MLWRKTLKN & 0.0944 & $\mathrm{NO}$ & 0.001155 & $\mathrm{NO}$ & 0.129977 & NO & 11879.35 & $\mathrm{NO}$ & -0.026 & NO \\
\hline 11 & MLWRKTSKN & 0.106 & NO & 0.000408 & NO & 0.087518 & NO & 13751.64 & NO & 0.03 & NO \\
\hline
\end{tabular}

373 Table 5: Experimental validation of peptides derived from exome sequencing analysis of breast cancer patients.

\section{Conclusion}


376 In this study, we have developed a deep learning-based model to predict the binding affinity of a peptide

377 with the HLA-A*02:01 receptors. The model employs both structure as well as sequence feature sets to

378 predict binding affinity. This approach is quite different from the previous structure only or sequence only

379 based approaches. Each of the methods has its advantage, however, combining features from both

380 domains can provide a better prediction of the binding affinity of the peptide with MHC. Our model not

381 only uses the information about the residue and their position in the peptide but also about the

382 interactions taking place at the atomic scale. Since, in the structure-based features, the whole peptide is

383 treated as a ligand, the residue-wise contribution towards peptide binding is lost. Thus, explicitly adding

384 the sequence-based features with the structure-based features would allow the network to learn from

385 the atomic interactions between the peptide-MHC as well as the different peptide residues contributing

386 towards binding. We tested 2 different architectures in the sequence module with 3 different sequence

387 feature sets. Although, there was not a very significant difference yet blosum62 encoding of the peptide

388 sequences showed the best performance in LSTM architecture of the sequence module along with the

389 structure module on the weekly benchmark dataset from IEDB. The combination of both structure and

390 sequence features increases the performance compared to those models with either of the features alone

391 as shown in model ablation analysis. Our model performed better than convMHC and HLAthena and is

392 comparable with the current state-of-the-art model, NetMHCpan4. Furthermore, the predictions made

393 by our model were consistent with the results from the molecular dynamics simulations and our model

394 performs well on the peptides from breast cancer patients validated experimentally. Due to the

395 involvement of intensive computation in structure modeling, the model was currently trained and tested

396 on HLA-A*02:01 only. In the near future, the model can be further extended to predict the binding

397 affinities of peptides to different alleles, a pan-alleles model. 
1. Castle JC, Kreiter S, Diekmann J, Lower M, van de Roemer N, de Graaf J, Selmi A, Diken M, Boegel $S$, Paret $C$ et al: Exploiting the mutanome for tumor vaccination. Cancer Res 2012, 72(5):1081-1091.

2. Banchereau J, Palucka K: Immunotherapy: Cancer vaccines on the move. Nat Rev Clin Oncol 2018, 15(1):9-10.

3. Yewdell JW, Bennink JR: Immunodominance in major histocompatibility complex class Irestricted T lymphocyte responses. Annu Rev Immunol 1999, 17:51-88.

4. Massimo Andreatta MN: Gapped sequence alignment using artificial neural networks: application to the MHC class I system. Bioinformatics 2016:511-517.

5. Jurtz V, Paul S, Andreatta M, Marcatili P, Peters B, Nielsen M: NetMHCpan-4.0: Improved Peptide-MHC Class I Interaction Predictions Integrating Eluted Ligand and Peptide Binding Affinity Data. J Immunol 2017, 199(9):3360-3368.

6. Vang YS, Xie X: HLA class I binding prediction via convolutional neural networks. Bioinformatics 2017, 33(17):2658-2665.

7. Sumanta Mukherjee CB, Nagasuma Chandra HLaffy: estimating peptide affinities for Class-1 HLA molecules by learning position-specific pair potentials. Bioinformatics 2016, 32(15):22972305.

8. Bordner AJ, Abagyan R: Ab initio prediction of peptide-MHC binding geometry for diverse class I MHC allotypes. Proteins 2006, 63(3):512-526.

9. Han Y, Kim D: Deep convolutional neural networks for pan-specific peptide-MHC class I binding prediction. BMC Bioinformatics 2017, 18(1):585.

10. Jianjun Hu ZL: DeepMHC: Deep Convolutional Neural Networks forHigh-performance peptideMHC Binding Affinity Prediction. 2017.

11. Yan Hu ZW, Hailin Hu, Fangping Wan, Lin Chen, Yuanpeng Xiong, Xiaoxia Wang, Dan Zhao, Weiren Huang, Jianyang Zeng: ACME: Pan-Specific peptide-MHC Class I Binding Prediction Through Attention-Based Deep Neural Networks Bioinformatics 2019:4946-4954.

12. Thammakorn Saethang $\mathrm{OH}$, Ingorn Kimkong, Vu Anh Tran, Xuan Tho Dang, Lan Anh T Nguyen, Tu Kien T Le, Mamoru Kubo, Yoichi Yamada, Kenji Satou: PAAQD: Predicting immunogenicity of MHC class I binding peptides using amino acid pairwise contact potentials and quantum topological molecular similarity descriptors. Journal of Immunological Methods 2013, 387(12):293-302.

13. Michelle P. Aranha CS, Omar Demerdash, Bogdan Czejdo, Jeremy C. Smith, Julie C. Mitchell: Prediction of peptide binding to $\mathrm{MHC}$ using machine learning with sequence and structurebased feature sets. Biochimica et Biophysica Acta (BBA) - General Subjects 2020, 1864(4).

14. Zheng L, Fan J, Mu Y: OnionNet: a Multiple-Layer Intermolecular-Contact-Based Convolutional Neural Network for Protein-Ligand Binding Affinity Prediction. ACS Omega 2019, 4(14):1595615965.

15. Sarkizova S, Klaeger S, Le PM, Li LW, Oliveira G, Keshishian H, Hartigan CR, Zhang W, Braun DA, Ligon KL et al: A large peptidome dataset improves HLA class I epitope prediction across most of the human population. Nat Biotechnol 2020, 38(2):199-209.

16. Vita R, Mahajan S, Overton JA, Dhanda SK, Martini S, Cantrell JR, Wheeler DK, Sette A, Peters B: The Immune Epitope Database (IEDB): 2018 update. Nucleic Acids Res 2019, 47(D1):D339D343. 
17. Kim Y, Sidney J, Buus S, Sette A, Nielsen M, Peters B: Dataset size and composition impact the reliability of performance benchmarks for peptide-MHC binding predictions. $B M C$ Bioinformatics 2014, 15:241.

18. Liu Z, Cui Y, Xiong Z, Nasiri A, Zhang A, Hu J: DeepSeqPan, a novel deep convolutional neural network model for pan-specific class I HLA-peptide binding affinity prediction. Sci Rep 2019, 9(1):794.

19. Wu J, Wang W, Zhang J, Zhou B, Zhao W, Su Z, Gu X, Wu J, Zhou Z, Chen S: DeepHLApan: A Deep Learning Approach for Neoantigen Prediction Considering Both HLA-Peptide Binding and Immunogenicity. Front Immunol 2019, 10:2559.

20. Helen M. Berman JW, Zukang Feng, Gary Gilliland, T. N. Bhat, Helge Weissig, Ilya N. Shindyalov, Philip E. Bourne: The Protein Data Bank Nucleic Acids Research 2000, 28(1):235-242.

21. Krivov GG, Shapovalov MV, Dunbrack RL, Jr.: Improved prediction of protein side-chain conformations with SCWRL4. Proteins 2009, 77(4):778-795.

22. Knapp B, Omasits U, Schreiner W: Side chain substitution benchmark for peptide/MHC interaction. Protein Sci 2008, 17(6):977-982.

23. Mark James Abraham TM, Roland Schulz, Szilárd Pálla, Jeremy C.Smith, Berk Hessa Erik Lindahl: GROMACS: High performance molecular simulations through multi-level parallelism from laptops to supercomputers. SoftwareX 2015, 1-2:19-25.

24. Henikoff S, Henikoff JG: Amino acid substitution matrices from protein blocks. Proc Natl Acad Sci U S A 1992, 89(22):10915-10919.

25. Vinyals $\mathrm{CZaSBaMHaBRaO}$ : Understanding deep learning requires rethinking generalization. arXiv:161103530 2017.

26. Hertz AKaJA: A Simple Weight Decay Can Improve Generalization. Advances in Neural Information Processing Systems 4 1992:950-957.

27. Moody JE: Note on generalization, regularization and architecture selection in nonlinear learning systems. Neural Networks for Signal Processing Proceedings of the 1991 IEEE Workshop 1991:1-10.

28. O'Donnell TJ, Rubinsteyn A, Bonsack M, Riemer AB, Laserson U, Hammerbacher J: MHCflurry: Open-Source Class I MHC Binding Affinity Prediction. Cell Syst 2018, 7(1):129-132 e124.

29. Phloyphisut P, Pornputtapong N, Sriswasdi S, Chuangsuwanich E: MHCSeqNet: a deep neural network model for universal MHC binding prediction. BMC Bioinformatics 2019, 20(1):270.

30. Lindorff-Larsen K, Piana S, Palmo K, Maragakis P, Klepeis JL, Dror RO, Shaw DE: Improved sidechain torsion potentials for the Amber ff99SB protein force field. Proteins 2010, 78(8):19501958.

31. Berk Hess HB, Herman J. C. Berendsen, Johannes G. E. M. Fraaije: Lincs: a linear constraint solver for molecular simulations. Journal of Computational Chemistry 1998, 18:1463-1472.

32. Berendsen WFVGaHJC: A Leap-frog Algorithm for Stochastic Dynamics. Molecular Simulation 1988, 1:173-185.

33. Harvey MJ, De Fabritiis G: An Implementation of the Smooth Particle Mesh Ewald Method on GPU Hardware. J Chem Theory Comput 2009, 5(9):2371-2377.

34. Hansen T, Myers N: Peptide induction of surface expression of class I MHC. Curr Protoc Immunol 2003, Chapter 18:Unit 1811.

35. Trolle T, Metushi IG, Greenbaum JA, Kim Y, Sidney J, Lund O, Sette A, Peters B, Nielsen M: Automated benchmarking of peptide-MHC class I binding predictions. Bioinformatics 2015, 31(13):2174-2181. 
36. Khan AR, Baker BM, Ghosh P, Biddison WE, Wiley DC: The structure and stability of an HLA$A * 0201 / o c t a m e r i c$ tax peptide complex with an empty conserved peptide- $\mathrm{N}$-terminal binding site. J Immunol 2000, 164(12):6398-6405.

37. Bouvier M, Wiley DC: Importance of peptide amino and carboxyl termini to the stability of MHC class I molecules. Science 1994, 265(5170):398-402.

38. Sharma AK, Kuhns JJ, Yan S, Friedline RH, Long B, Tisch R, Collins EJ: Class I major histocompatibility complex anchor substitutions alter the conformation of $\mathrm{T}$ cell receptor contacts. J Biol Chem 2001, 276(24):21443-21449.

39. Ruppert J, Sidney J, Celis E, Kubo RT, Grey HM, Sette A: Prominent role of secondary anchor residues in peptide binding to HLA-A2.1 molecules. Cell 1993, 74(5):929-937.

40. Robert A. Colbert SLR-J, Andrew J. McMichael, Jeffrey A. Frelinger: Differences in peptide presentation between B27 subtypes: The importance of the P1 side chain in maintaining high affinity peptide binding to $B \star 2703$. Immunity 1994,1 (2):121-130.

41. Guterres H, Im W: Improving Protein-Ligand Docking Results with High-Throughput Molecular Dynamics Simulations. J Chem Inf Model 2020, 60(4):2189-2198.

42. Rashmi Kumari RK, Open Source Drug Discovery Consortium and Andrew Lynn: g_mmpbsa-A GROMACS Tool for High-Throughput MM-PBSA Calculations. Journal of Chemical Information and Modelling 2014.

\section{Declarations:}

\section{Acknowledgments:}

511 We thank Liangzhen Zheng for providing his valuable inputs that helped in optimizing the deep learning

512 models developed in this study.

\section{Funding:}

514 This research is supported by MOE Tier 1 Grant RG146/17 awarded to Yuguang Mu; National Research

515 Foundation (NRF) Singapore through an NRF Fellowship awarded to M.J.F (NRF-NRFF2012-054); NTU

516 start-up funds awarded to M.J.F.; RNA Biology Center at the Cancer Science Institute of Singapore, NUS,

517 as part of funding under the Singapore Ministry of Education Academic Research Fund Tier 3 awarded to

518 Daniel Tenen (MOE2014-T3-1-006); National Research Foundation Singapore and the Singapore Ministry 
519 of Education under its Research Centres of Excellence initiative. The computing facility was supported by

520 the National Supercomputing Centre, Singapore (https://www.nscc.sg).

\section{Availability of data and its materials:}

522 OnionMHC model and its source code, together with a usage guide, the dataset used for training as well

523 as weekly benchmark data used for independent evaluation are freely available on GitHub at

524 https://github.com/shikhar249/OnionMHC. The binding affinity data used for training is also provided in

525 Additional File 4.

\section{Authors' Affiliations:}

527 Cancer Science Institute, National University of Singapore, 14 Medical Drive, Singapore, 117599

528 Sambhavi Animesh and Melissa Fullwood

529 School of Biological Sciences, Nanyang Technological University, 60 Nanyang Drive, Singapore, 637551

530 Shikhar Saxena, Melissa Fullwood, and Yuguang Mu

\section{Authors' Contributions:}

532 SS and YM designed the study. SS developed and analyzed the model. SA performed the experimental

533 validation of peptides. YM and MF provided overall supervision. SS and YM wrote the manuscript with

534 assistance from other authors. All authors approved the final manuscript.

\section{Corresponding author:}

536 Correspondence to Yuguang $\mathrm{Mu}$

\section{Ethics approval and consent to participate:}

538 Not applicable

\section{Consent for publication:}


540 Not applicable

\section{Competing interests:}

542 The authors declare no competing interests. 
a)

\section{\begin{tabular}{lll|l|l|l|l|l|l|l|l|l|l|l|l|l|l|l|l|l|l} 
A & C & D & E & F & G & H & I & K & L & M & N & P & Q & R & S & T & V & W & Y
\end{tabular}}

\begin{tabular}{lllllllllllllllllllllllllll|}
$A$ & 0 & 1 & 0 & 0 & 0 & 0 & 0 & 0 & 0 & 0 & 0 & 0 & 0 & 0 & 0 & 0 & 0 & 0 & 0 & 0 & 0 \\
\hline
\end{tabular}

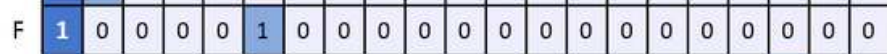

\begin{tabular}{llllllllllllllllllllllll|}
\hline & 2 & 0 & 0 & 0 & 0 & 0 & 0 & 0 & 0 & 0 & 0 & 0 & 0 & 0 & 0 & 0 & 0 & 0 & 1 & 0 & 0 \\
\hline
\end{tabular}

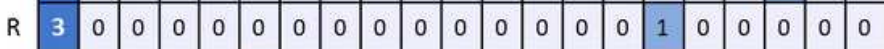

F 4 \begin{tabular}{lllllllllllllllllllllllll|}
\hline & 0 & 0 & 0 & 0 & 1 & 0 & 0 & 0 & 0 & 0 & 0 & 0 & 0 & 0 & 0 & 0 & 0 & 0 & 0 & 0 \\
\hline
\end{tabular}

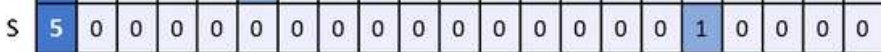

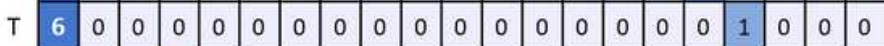

D 7 \begin{tabular}{lllllllllllllllllllllllllll|}
\hline & 0 & 0 & 1 & 0 & 0 & 0 & 0 & 0 & 0 & 0 & 0 & 0 & 0 & 0 & 0 & 0 & 0 & 0 & 0 & 0 \\
\hline
\end{tabular}

K 8 \begin{tabular}{llllllllllllllllllllllllll|}
8 & 0 & 0 & 0 & 0 & 0 & 0 & 0 & 0 & 1 & 0 & 0 & 0 & 0 & 0 & 0 & 0 & 0 & 0 & 0 & 0 \\
\hline
\end{tabular}

b) \begin{tabular}{l|l|l|l|l|l|l|l|l|l|l|l|l|l|l|l|l|l|l|l|l|l|} 
A & R & N & D & C & Q & E & G & H & I & L & K & M & F & P & S & T & W & Y & V \\
\hline
\end{tabular}

\begin{tabular}{lllllllllllllllllll|l|l|l|l|l|l|l|}
$\mathrm{A}$ & 0 & 4 & -1 & -2 & -2 & 0 & -1 & -1 & 0 & -2 & -1 & -1 & -1 & -1 & -2 & -1 & 1 & 0 & -3 & -2 & 0 \\
\hline
\end{tabular}

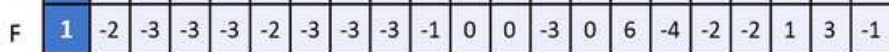

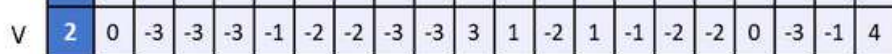

\begin{tabular}{llllllllllllllllllllllllllll|}
\hline $\mathrm{R}$ & 3 & -1 & 5 & 0 & -2 & -3 & 1 & 0 & -2 & 0 & -3 & -2 & 2 & -1 & -3 & -2 & -1 & -1 & -3 & -2 & -3 \\
\hline
\end{tabular}

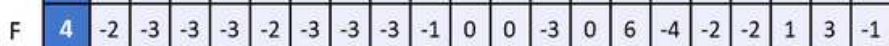

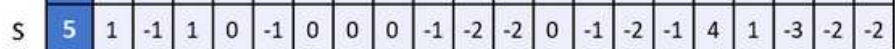

T \begin{tabular}{llllllllllllllllllllllllllll|}
\hline & 6 & 0 & -1 & 0 & -1 & -1 & -1 & -1 & -2 & -2 & -1 & -1 & -1 & -1 & -2 & -1 & 1 & 5 & -2 & -2 & 0 \\
\hline
\end{tabular}

D \begin{tabular}{llllllllllllllllllllllllllll}
\hline & -2 & -2 & 1 & 6 & -3 & 0 & 2 & -1 & -1 & -3 & -4 & -1 & -3 & -3 & -1 & 0 & -1 & -4 & -3 & -3 \\
\hline
\end{tabular}

\begin{tabular}{llllllllllllllllllllllllllllll|}
\hline $\mathrm{K}$ & 8 & -1 & 2 & 0 & -1 & -3 & 1 & 1 & -2 & -1 & -3 & -2 & 5 & -1 & -3 & -1 & 0 & -1 & -3 & -2 & -2 \\
\hline
\end{tabular} c) $\quad A: 1, C: 2, D: 3, E: 4, F: 5 \ldots V: 18, W: 19, Y: 20$

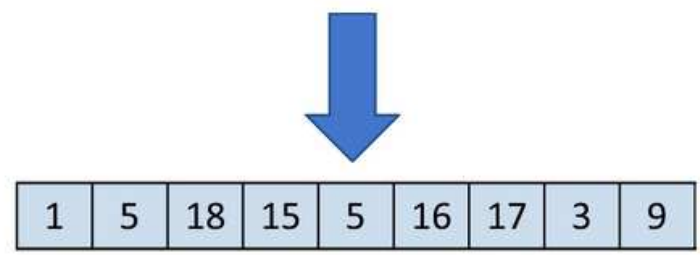

Embedding Vector

Embedding Matrix

\section{Figure 1}

Illustration of encoding of peptide AFVRFSTDK with three different encoding techniques used in this study a) In one-hot encoding, each position is represented as a 20-dimensional vector with the value of corresponding residue kept as 1 and remaining 19 residues as 0 b) In BLOSUM62 encoding, each position is represented by the vector corresponding to the residue from the BLOSUM62 matrix c) In embedding vector, each residue is assigned an integer value and the peptide is represented as embedding vector formed out of those integer values.
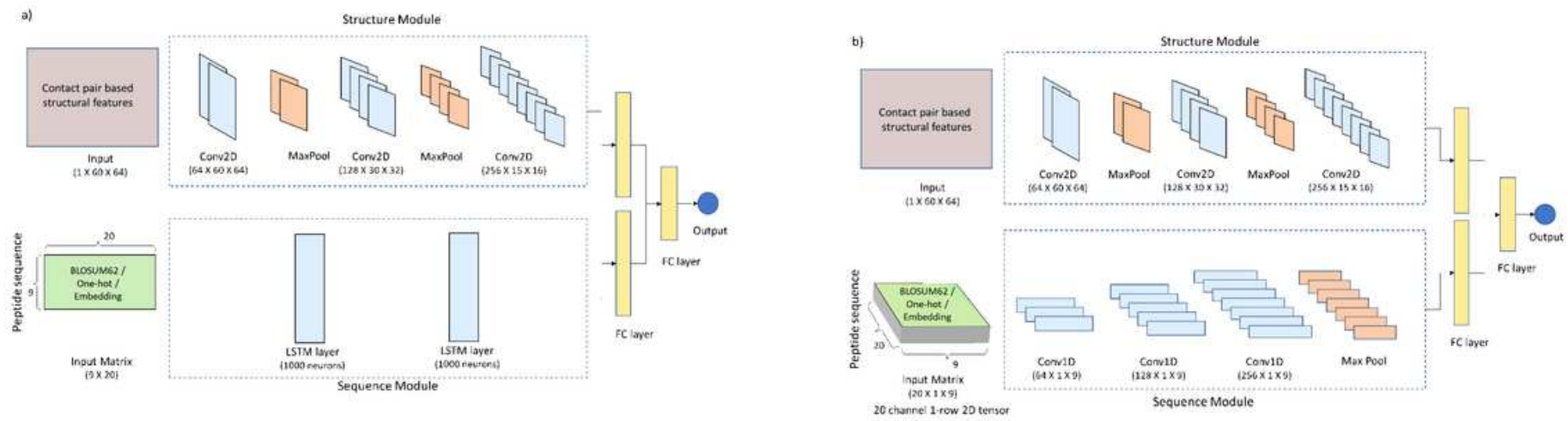
Architectures tested in this study with structure module based on Convolutional Neural Network: a) Long Short-Term Memory based neural network was used in sequence module with two Istm layers of 1000 neurons each and its output is combined with that of structure module using Fully Connected (FC) layer b) Convolutional Neural Network-based neural network was used in sequence module with three 1-D convolutional layers (of filter size $64,128,256$ ), followed by maximum pooling and its output is also combined with that of structure module using Fully Connected (FC) layer

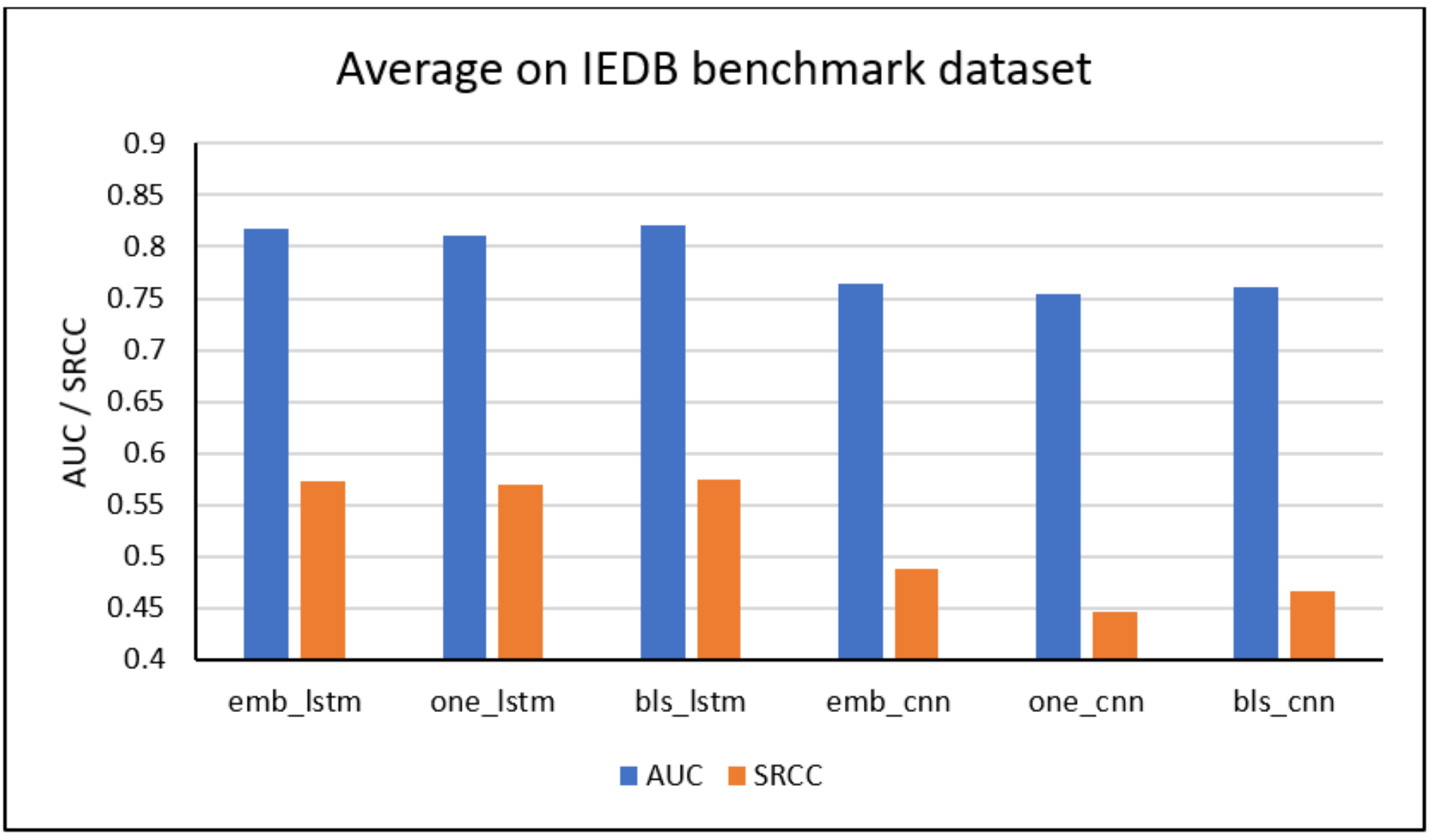

Figure 3

Performance evaluation of the six generated models on the IEDB benchmark dataset 


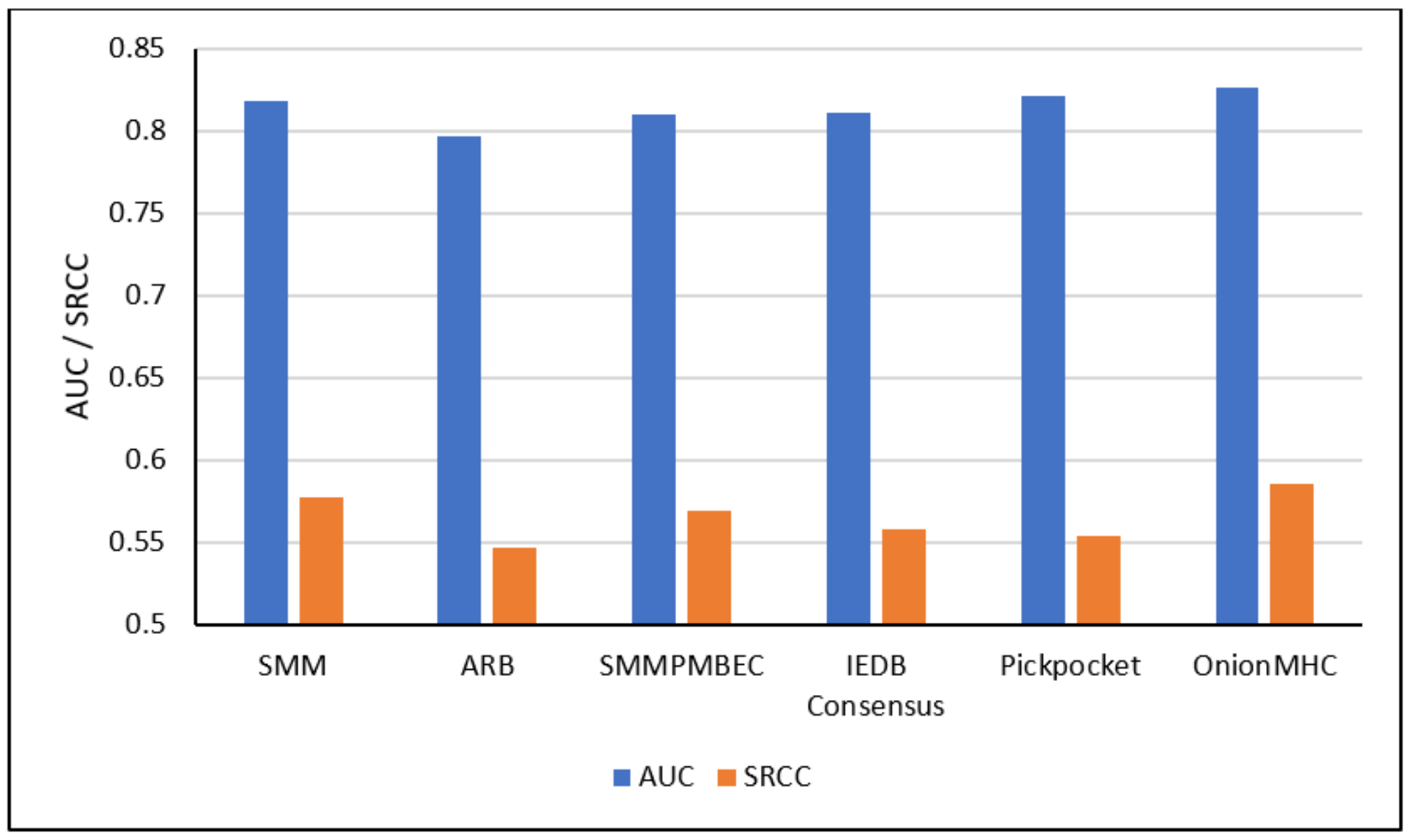

Figure 4

Performance comparison of OnionMHC with different prediction algorithms (Trolle et. al 2015) on the most recent IEDB benchmark dataset, measured in terms of Spearman Rank Correlation Coefficient (SRCC) and area under receiver operating curve (AUC) 
a)

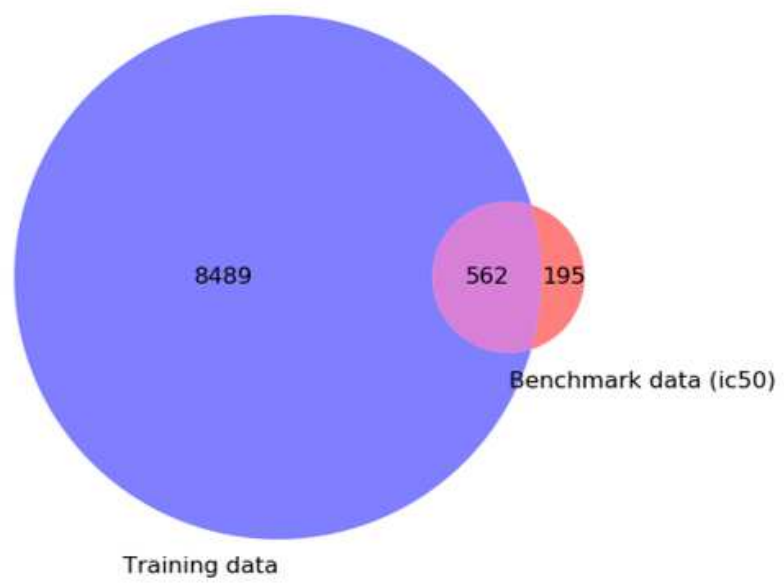

b)

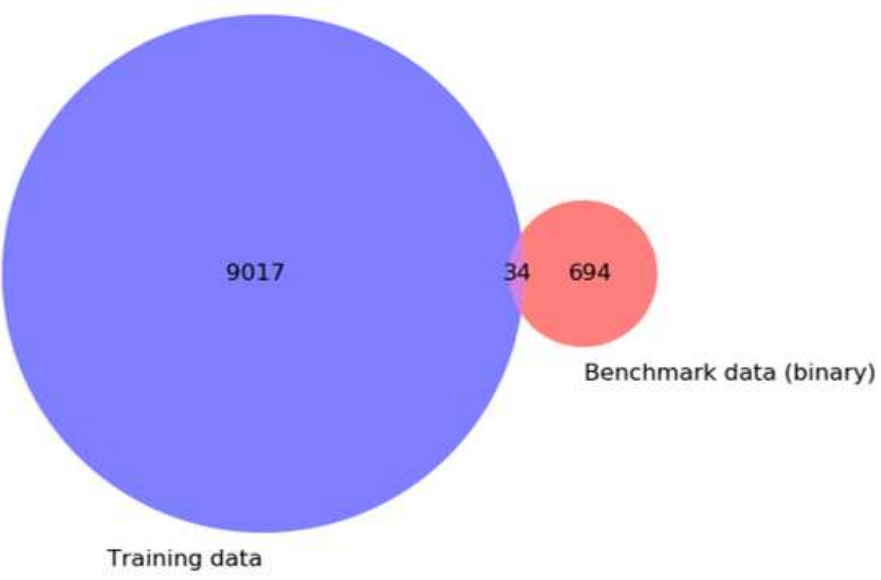

c)

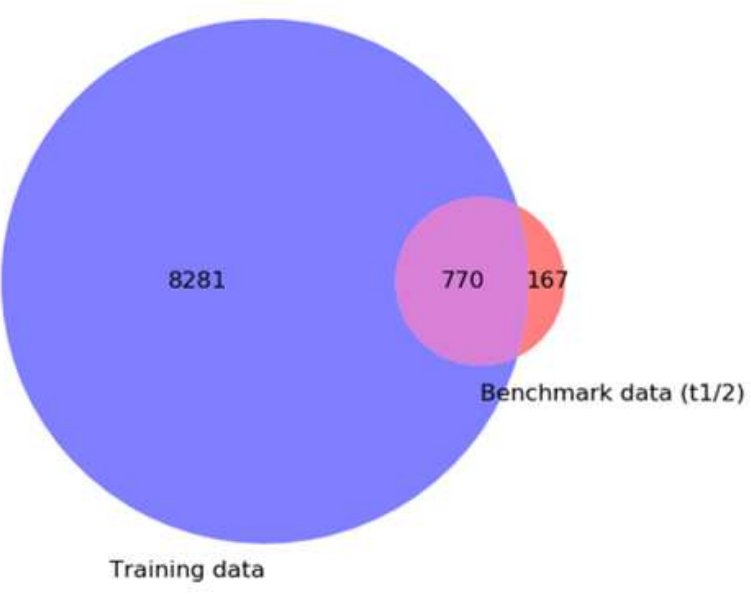

\section{Figure 5}

Overlapping peptides between the training data and benchmark data with measurement value: a) binary; b) ic50; c) half-life (t1/2) 

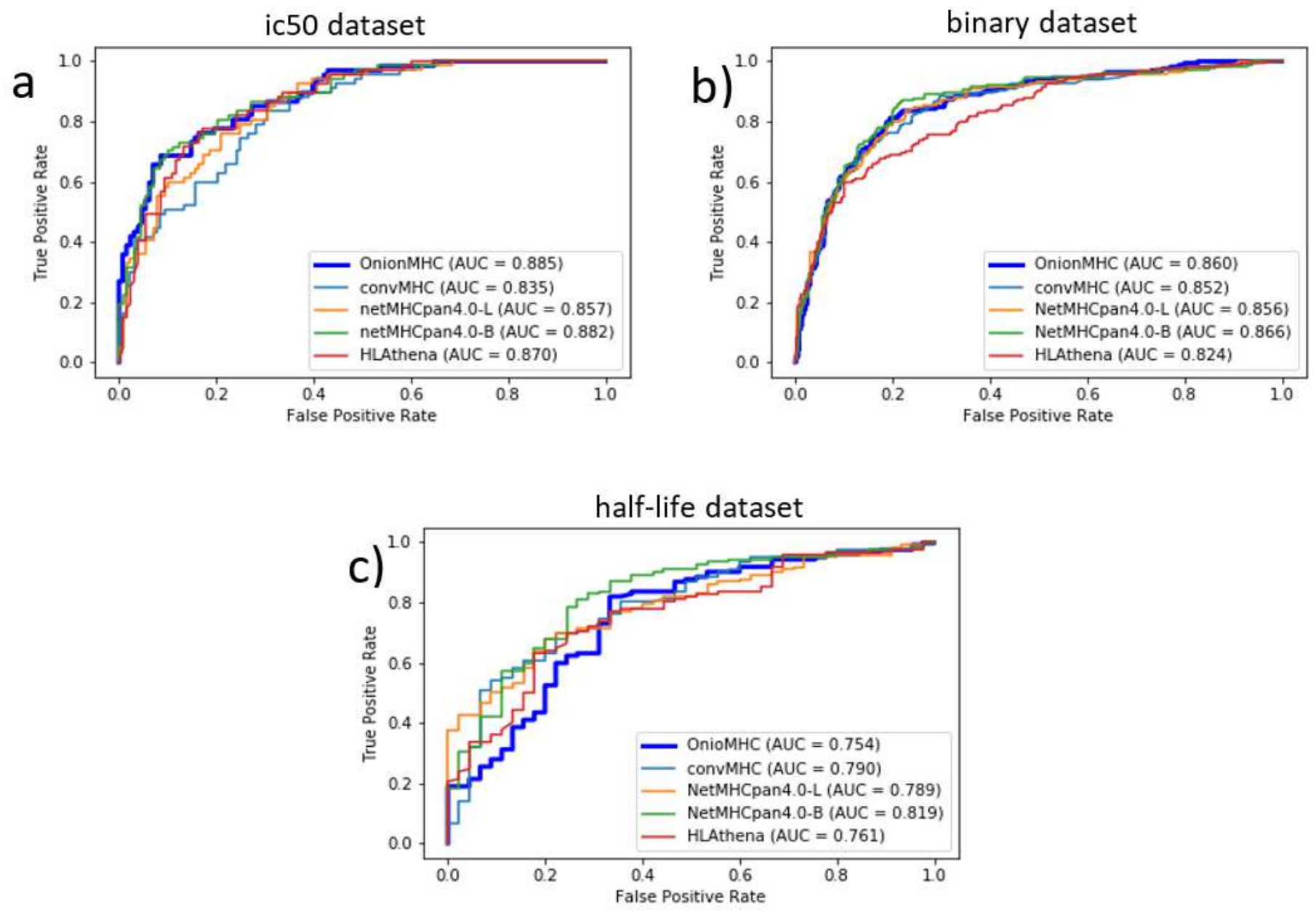

\section{Figure 6}

Area under the receiver operating curves for the non-overlapping peptides a) ic50 dataset (195 peptides) b) binary dataset (694 peptides) c) half-life dataset (167 peptides) 


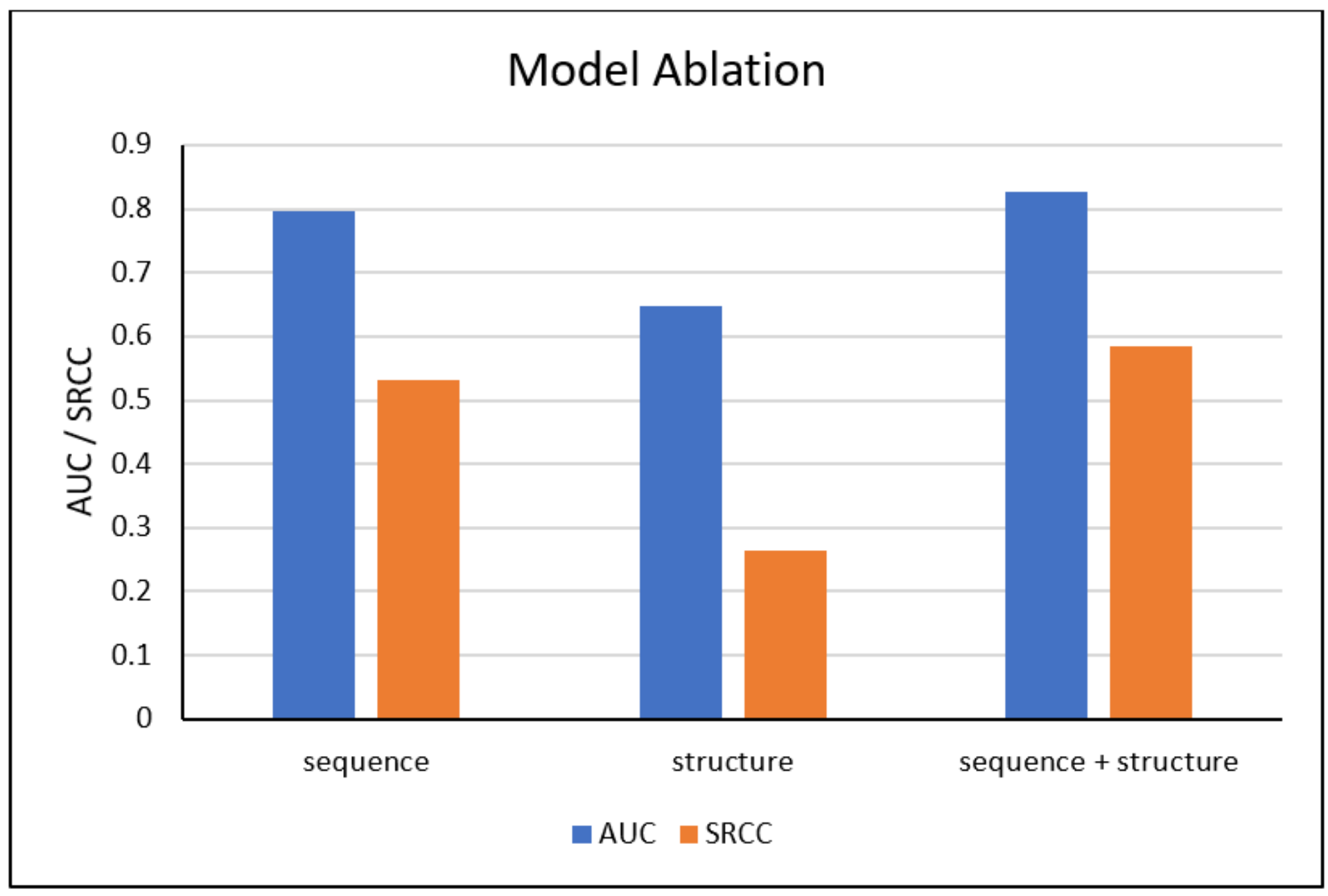

Figure 7

Model Ablation analysis of OnionMHC showing the individual performance of sequence and structure module on IEDB benchmark dataset 


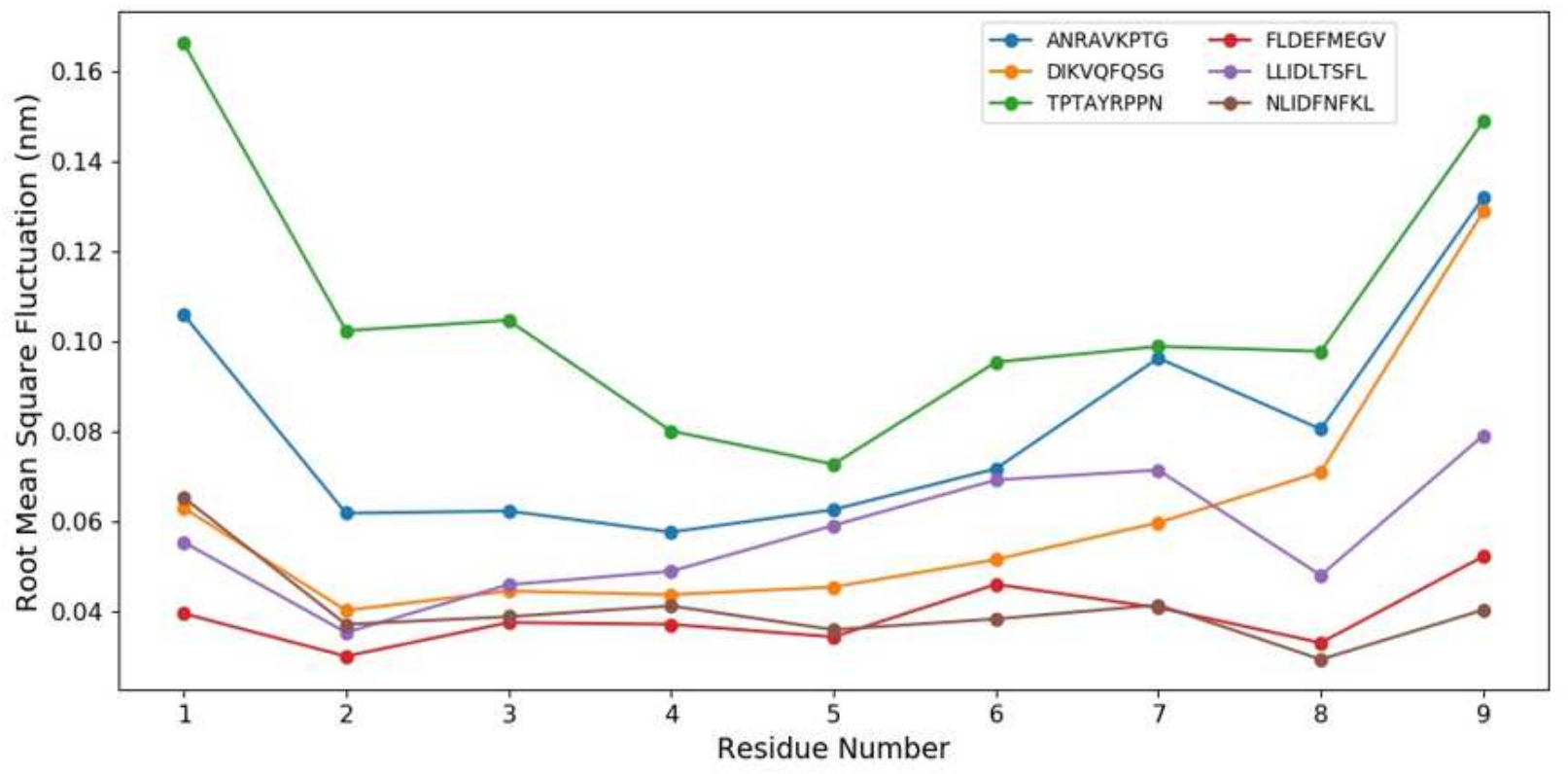

Figure 8

Root Mean Square Fluctuation $(\mathrm{nm})$ of the residues in the peptide in the Molecular Dynamics simulation.

\section{Supplementary Files}

This is a list of supplementary files associated with this preprint. Click to download.

- AdditionalFile1.docx

- AdditionalFile2.xIsx

- AdditionalFile3.xlsx

- AdditionalFile4.xlsx 